\title{
1 Bacterial supergroup specific "Cost" of Wolbachia infections in
}

\section{Nasonia vitripennis}

3 Alok Tiwary ${ }^{1}$, Rahul Babu $^{1,2}$, Ruchira Sen $^{3}$, Rhitoban Raychoudhury $^{1^{*}}$

4

5

6

\section{5}

1. Department of Biological Sciences, Indian Institute of Science Education and Research (IISER) Mohali, Knowledge City, Sector 81, SAS Nagar, Manauli, PO 140306, Punjab, India.

2. Zoological Survey of India, Kolkata, West Bengal-700053, India

3. Guru Gobind Singh College, Sector 26, Chandigarh-160019, India.

*Corresponding author: Rhitoban Raychoudhury, Department of Biological Sciences, Indian Institute of Science Education and Research (IISER) Mohali, Sector 81, Knowledge City, SAS Nagar, Manauli, PO 140306 (Punjab), India

Email: rhitoban@iisermohali.ac.in

Key Words: Wolbachia, Nasonia, life-history traits, Quantitative PCR, Mann-Whitney U, Prospermatogeny, Cytoplasmic incompatibility 
The maternally-inherited endosymbiont, Wolbachia, is known to alter the reproductive biology of its arthropod hosts for its benefit and can induce both positive and negative fitness effects in many hosts. Here we describe the effects of the maintenance of two distinct Wolbachia infections, one each from supergroups $A$ and $B$, on the parasitoid host Nasonia vitripennis. We compare the effect of Wolbachia infections on various traits between the uninfected, single A

25 infected, single B infected, and the double infected strains with their cured versions. Contrary

26 to the previous reports, our results suggest that there is a significant cost associated with the

27 maintenance of Wolbachia infections where traits like family size, fecundity, longevity, and

28 rates of male copulation are compromised in Wolbachia infected strains. The double infected

29 and supergroup B infection strains show higher Wolbachia titer than supergroup A. The double infected Wolbachia strain has the most detrimental impact on the host as compared to single infections. Moreover, there is a supergroup-specific negative impact on these wasps as the supergroup B infections elicit the most pronounced negative effects. These findings raise important questions on the mechanism of survival and maintenance of these reproductive parasites in arthropod hosts. 


\section{Introduction}

40

41

42

43

44

45

46

47

Wolbachia are maternally-inherited, obligatory intracellular endosymbionts of the order Rickettsiales (Hertig et al., 1927) that are widely found in arthropods and filarial nematodes (Bandi et al. 1992; Rousset et al. 1992; Weinert et al., 2015). These bacteria often alter host reproductive biology with mechanisms like male-killing, feminization, parthenogenesis, and cytoplasmic incompatibility (CI), to enhance their own transmission (Werren et al., 2008). While $\mathrm{Cl}$ leads to an increase in the number of infected individuals in the population, male-killing, and feminization shifts the offspring sex ratio towards females, which is the transmitting sex for Wolbachia. Thus, Wolbachia increases the fitness of the infected hosts as it increases its own rate of transmission. While these cases highlight a parasitic effect of Wolbachia, there are several examples where no such effect is discernible (Hoffmann et al., 1996). Moreover, there are also examples where Wolbachia has now become a mutualist and offers specific and quantifiable benefits to its host. One such example of an obligate mutualism with Wolbachia has been reported in Asobara tabida where females cured of Wolbachia are unable to produce mature oocytes (Dedeine et al., 2001). Such examples of arthropod-Wolbachia mutualism have now been reported from various arthropod taxa (Pike \& Kingcombe, 2009; Miller et al., 2010). This shift from parasitic to mutualistic effect can also happen in facultative associations as seen in Drosophila simulans, where within a span of just two decades, Wolbachia has evolved from a parasite to a mutualist (Weeks et al., 2007). However, for the majority of Wolbachia-host associations, no such reports of mutualistic benefits exist and instead reveal many negative effects on the host. In addition to reproductive traits, many other life-history traits like 
longevity and developmental time are also known to be compromised. A review of such negative effects of Wolbachia on hosts where $\mathrm{Cl}$ is prevalent is presented in Table 1 . In Trichogramma kaykai and T. deion, the infected (thelytokous) line shows reduced fecundity and adult emergence rates than the antibiotically cured (arrhenotokous) lines (Hohmann et al., 2001; Tagami et al., 2001). Leptopilina heterotoma, a Drosophila parasitoid, has adult survival rates, fecundity, and locomotor performance, of both sexes, severely compromised in Wolbachia infected strains (Fleury et al., 2000). Preimaginal disc mortality has been observed in both sexes of insecticide-resistant Wolbachia-infected strains of Culex pipiens (Duron et al., 2006). Wolbachia infections can also result in a range of behavioral changes and altered phenotypes in Aedes aegypti (Turley et al., 2009).

These negative effects of Wolbachia on their hosts are not unexpected. The presence of

71 bacteria within a host entails sharing of nutritional and other physiological resources

72 (Kobayashi \& Crouch, 2009; Whittle et al., 2021), especially with Wolbachia as they are obligate endosymbionts and cannot survive without cellular resources derived from their hosts (Foster et al., 2005., Slatko et al., 2010). For example, Wolbachia is known to compete with the host for key resources like cholesterol and amino acids in A. aegypti (Caragata et al., 2014). The precise molecular mechanisms of many of these negative effects have not been ascertained and are

77 generally ascribed to partitioning off of host nutrients for its benefit, but what is clear is that Wolbachia infections can impose severe nutritional demands on their hosts (Ponton et al., 2014). However, it is also known that Wolbachia can elicit antipathogenic responses from their

80 hosts where the host resistance or tolerance to the infection increases (Zug \& Hammerstein, 81 2015). For example, Wolbachia can utilize the Immune deficiency (IMD) and Toll pathways (Pan 
et al., 2018) and increased ROS (Reactive Oxygen Species) levels in Wolbachia-transfected $A$. aegypti mosquitoes, inhibiting the proliferation of the Dengue virus (Pan et al., 2012) as well as against Drosophila C viruses (Teixeira et al., 2008). Such immune responses require additional allocation of resources, which can further affect other physiological traits of the host. This concept of a "cost of immunity" is well-established and suggests a trade-off between immunity and other life-history traits (Zuk \& Stoehr, 2002). For example, elevated ROS levels negatively affect many host traits like longevity, and fecundity (Dowling \& Simmons, 2009; Monaghan et al., 2009; Selman et al., 2012; Moné et al., 2014). Thus, there is sufficient evidence to conclude that Wolbachia can have substantial negative effects on the overall fitness of its host.

In this study, we investigate what, if any, are the negative effects on the wasp N. vitripennis due to its two resident $\mathrm{Cl}$ inducing Wolbachia infections? N. vitripennis, being cosmopolitan, has been used to study Wolbachia distribution, acquisition, spread, and Wolbachia-induced reproductive manipulations (Werren et al., 2008; Landmann, 2019). However, the effect of the endosymbiont on the host life-history traits of this wasp remains poorly understood with conflicting reports. In some $N$. vitripennis strains, Wolbachia was reported to be the cause of enhanced fecundity (Stolk \& Stouthamer, 1996), but a similar effect was not observed in some other strains (Bordenstein \& Werren, 2000). N. vitripennis harbor two Wolbachia supergroup infections, one each from supergroup A and supergroup B (Perrot-Minnot et al., 1996), and the presence of these two infections have been found in all strains of $N$. vitripennis from continental North America to Europe (Raychoudhury et al., 2010), indicating that it has reached fixation across the distribution of its host. The two Wolbachia in N. vitripennis together cause complete $\mathrm{Cl}$, but single infections of supergroup $\mathrm{A}$ Wolbachia causes incomplete $\mathrm{Cl}$ while 
104

105

106

107

108

109

110

111

112

113

114

115

116 117 such negative effects only for a few of those traits. By testing for differential titer of bacteria by

supergroup B infections still show complete Cl (Perrot-Minnot et al., 1996). In this study, we investigate the effects of Wolbachia infections in a new strain of $N$. vitripennis recently obtained from the field. This strain, like other N. vitripennis strains, has two Wolbachia infections, one each from the supergroup A and B. Sequencing of the five alleles from the wellestablished multi-locus strain typing system (Baldo et al., 2006) reveal no sequence dissimilarity across the two infections (Prazapati, personal communication) indicating this new strain is also infected by the same or very similar Wolbachia strains that are present across the distribution of $N$. vitripennis (Raychoudhury et al., 2010b). To compare supergroup specific effects, these two infections have been separated into single infected strains. A comparative analysis between the double infected, supergroup A infected, supergroup B infected and uninfected strains revealed a consistent pattern of decreased longevity, quicker sperm depletion and reduced family size for infected individuals. While supergroup B infection has a more pronounced negative effect on most of the traits investigated, supergroup A infection show qRT-PCR, we also show a higher density of supergroup B and double infected strains across the different developmental stages of $N$. vitripennis males and females as compared to the supergroup A infected ones.

\section{Materials and Methods}

\section{N. vitripennis strains used, their Wolbachia infections, and strain nomenclature}

The N. vitripennis strains used NV PU-14 were obtained from Mohali, Punjab, India, in 2015. NV PU-14 was cured of Wolbachia by feeding the females with $1 \mathrm{mg} / \mathrm{ml}$ tetracycline in $10 \mathrm{mg} / \mathrm{ml}$ 
125 sucrose solution for at least two generations (Breeuwer \& Werren, 1990). The curing was

126 confirmed by PCR using supergroup-specific ftsZ primers (Baldo et al., 2006), and Cl crosses

127 between the infected and uninfected strains. NV PU-14 also served as the source strain for

128 separating the two Wolbachia infections into single A and single B infected strains.

129 To separate the strains, we utilized relaxed selection on the females by repeatedly mating them

130 with uninfected males which were obtained by antibiotic curing of the same NV PU-14 strain.

131 Uninfected males do not have any sperm modification by Wolbachia which results in the

132 removal of any selection pressure on the females to maintain their Wolbachia infections.

133 Repeated mating with uninfected males was continued for ten generations till some of the

134 progenies were found to be infected with either single A or single B supergroup infections. The

135 single infection status of these strains was confirmed by using supergroup-specific fts $Z$ gene

136 PCR primers (Baldo et al., 2006).

137 The preferred method of nomenclature of Nasonia strains and their Wolbachia infections

138 includes information on supergroups as well as the host genotype. For example, [wNvitA

$139 w$ NvitB]V-PU14 indicates that the host species is N. vitripennis, with NV-PU14 as the host

140 genotype, which has two Wolbachia infections, one each from supergroup A and supergroup B.

141 However, since we used only N. vitripennis strains in this study, the nomenclature has been

142 simplified by removing the species name. For example, the same strain will now be denoted as

$143 w A w B(P U)$, and when cured of these infections, as $0(P U)$. The single Wolbachia-infected strains

144 used were designated as $w \mathrm{~A}(\mathrm{PU})$ for the supergroup $\mathrm{A}$-infected strain while $w \mathrm{~B}(\mathrm{PU})$ for the

145 supergroup B-infected strain. As the cured O(PU) lines were in culture for three years, many of 
146 the infected lines were cured again to obtain 'recently cured' lines to minimize the effects of

147 any host divergence that might have accumulated within them. These 'recently cured' lines

148 were named $\mathrm{O}(w \mathrm{~A} \mathrm{PU}), \mathrm{O}(w \mathrm{~B} \mathrm{PU})$, and $\mathrm{O}(w \mathrm{~A} w \mathrm{~B} \mathrm{PU})$. All these wasp strains were raised on

149 Sarcophaga dux fly pupae with a generation time of $14-15$ days at $25^{\circ} \mathrm{C}, 60 \%$ humidity, and a

150 continuous daylight cycle.

151 Sequential mating and sperm depletion of the males

152 To test the effect of Wolbachia on male reproductive traits like mating ability, individual males

153 were assayed for the number of copulations it can perform, as well as sperm depletion. As $N$.

154 vitripennis is haplodiploid, every successful mating will result in both female and male

155 progenies while an unsuccessful one will result in the production of exclusively male progenies.

156 The males used were obtained from virgin females hosted with one fly pupa for 24 hours and

157 were not given any external sources of nutrition (usually a mixture of sucrose in water) before

158 the experiment. Each male was then mated sequentially with virgin females from the same

159 strain. At the first sign of a male not completing the entire mating behavior (Jachmann \&

160 Assem, 1996), it was given a rest for half an hour and was subjected to mating again until it

161 stopped mating altogether. The mated females were hosted after a day with one fly pupa for 24

162 hours. The females were then removed and the offspring were allowed to emerge and then

163 counted. The average number of copulations and the number of copulations before sperm

164 depletion were compared using the Mann-Whitney $U$ test with a significance level of 0.05.

165 Host longevity, family size, and fecundity 
166 To test whether the presence of Wolbachia has any influence on longevity, emerging wasps of

167 both the sexes were kept individually in ria vials at $25^{\circ} \mathrm{C}$, without any additional nutrition.

168 Survival following emergence was measured by counting the number of dead individuals every

1696 hours. Kaplan-Meier analysis followed by Log Rank Statistics was used to identify differences

170 between strains with a significance level of 0.05 .

171 To test for the effect of Wolbachia infections on the adult family size of virgin and mated

172 females, each female was sorted at the pupal stage and separated into individual ria vials. To

173 enumerate the brood size of mated females, some of these virgins were offered single males

174 from the same strain and observed till mating was successful. All the females were then hosted

175 individually with one fly pupa for 24 hours. These were kept at $25^{\circ} \mathrm{C}$ for the offspring to emerge

176 which were later counted for family size by randomizing the ria vials in a double-blind assay.

177 The differences were compared using the Mann-Whitney $U$ test with a significance level of

$178 \quad 0.05$.

179 To investigate if Wolbachia affects the female fecundity, emerged females were hosted with 180 one host for 24 hours. The host pupa was placed in a foam plug so that only the head portion of 181 the pupa was exposed and available for the females to lay eggs. They were removed after 24 182 hours and the eggs laid were counted under a stereomicroscope (Leica M205 C). The 183 differences in fecundity were compared using the Mann-Whitney $U$ test with a significance 184 level of 0.05.

185 Estimation of relative density of Wolbachia infections across different 186 developmental stages of $N$. vitripennis 
187 To collect the different developmental stages, females were hosted for 4 hours, (instead of 24

188 hours in the previous experiments), with one host to narrow down the developmental stages of 189 the broods. The larval and pupal stages (from Day 3 to Day 13 for males and from Day 8 to Day

19014 for females) were collected every 24 hours. Larval stages for females were not done to avoid 191 any DNA contamination from the males as the two sexes are virtually indistinguishable at these 192 stages. Three replicates of ten larvae or pupae from the three strains, $w A(P U), w B(P U)$, and $193 w A w B(P U)$, were collected for each developmental stage. DNA extraction was done using the 194 phenol-chloroform extraction method, where samples were crushed in $200 \mu$ of $0.5 \mathrm{M}$ Tris195 EDTA buffer with $1 \%$ sodium dodecyl sulfate (SDS) and $2 \mu \mathrm{l}$ of $22 \mathrm{mg} / \mathrm{ml}$ Proteinase $\mathrm{K}$ and 196 incubated overnight at $37^{\circ} \mathrm{C}$. DNA was purified with buffer saturated phenol and chloroform197 isoamyl alcohol solution (24:1) and precipitated overnight with isopropanol at $-20^{\circ} \mathrm{C}$. The 198 precipitated DNA pellet was dissolved in $60 \mu$ nuclease-free water. The DNA concentration of 199 the samples was measured using the Nanodrop $2000^{\circledR}$ spectrophotometer (Thermo Scientific).

200 The concentrations of all the samples were normalized to $200 \mathrm{ng} / \mu \mathrm{l}$ across the different male 201 and female developmental stages to be used for quantitative PCR. CFX96 C1000 ${ }^{\circledR}$ Touch Real202 time qRT-PCR machine (BioRad) was used to assay the relative density of Wolbachia across the 203 strains. Amplification was done for the Wolbachia hcpA gene (Forward Primer: 5'204 CTTCGCTCTGCTATATTTGCTGC-3', Reverse Primer: 5'-CGAATAATCGCAACCGAACTG-3'1. The primers were tested to amplify both the Wolbachia supergroup A and B strains. Nasonia S6K 206 was used as the control gene (Bordenstein and Bordenstein, 2011). The total reaction volume 207 contained $5 \mu$ l of iTaq Universal SYBR ${ }^{\circledR}$ Green supermix (BIO-RAD), $0.05 \mu$ l each of $10 \mu \mathrm{M}$ of 208 forward and reverse primers, and $200 \mathrm{ng}$ of template DNA for a total volume of $10 \mu \mathrm{l}$ for each 
209

210

211

212

213

214

215

216

217

218

219

220

221 Results

222

223

224

225

226

227

228

reaction. Uninfected $N$. vitripennis DNA was used as negative control while DNase-free water was used as non-template control. Reaction conditions included an initial denaturation step of $95^{\circ} \mathrm{C}$ for 3 minutes followed by 39 cycles of $95^{\circ} \mathrm{C}$ for 10 seconds, annealing, and amplification at $52^{\circ} \mathrm{C}$ for 30 seconds for 40 cycles. All the reactions were performed in triplicates and included a melt curve to check for non-specific amplification. The relative density of Wolbachia was estimated by calculating the mean delta threshold cycle $\left(\Delta \mathrm{C}_{\mathrm{q}}\right)$, using the formula:

$$
\Delta \mathrm{C}_{\mathrm{q}}=1 / 3 \sum_{j=1}^{3}\left[1 / 3 \sum_{i=1}^{3} h c p A-1 / 3 \sum_{i=1}^{3} S 6 K\right]
$$

where $\mathrm{i}=$ number of technical replicates and $\mathrm{j}=$ number of biological replicates.

1/ $\Delta \mathrm{Cq}$ was calculated and plotted to show the Wolbachia density across different developmental stages of males and females. The relative Wolbachia density was compared across the three different strains using the Kruskal Wallis $H$ test. Mann Whitney $U$ test was used to compare two different groups with a significance level of 0.05 .

\section{The presence of Wolbachia reduces the lifespan of both males and females}

Wolbachia can compete with the host for available nutrition which can increase nutritional stress resulting in a shortened lifespan of many hosts (McMeniman et al., 2009; Caragata et al., 2014). Therefore, we first investigated the effect of Wolbachia infections on the survival of both male and female wasps. As Fig. 1(A), indicates, there is a significant difference in the longevity of the infected males across the three infection types. The double-infected strain, $w A w B(P U)$, starts to die-off first and has a significantly shorter life span compared to the two single 
229 infected strains $\left\{\right.$ Log Rank Test, $\chi^{2}=16.8, p<0.001$ for $w A(P U)$ and $\chi^{2}=33.9, p<0.001$ for $w \mathrm{~B}(\mathrm{PU})\}$. Males from the uninfected strain, $\mathrm{O}(\mathrm{PU})$, lived the longest and showed significantly longer lifespan compared to all the other infected strains $\left\{\right.$ Log Rank Test: $\chi^{2}=76.3, p<0.001$ for $w A w B(P U) ; \chi^{2}=33.0, p<0.001$ for $w A(P U)$ and $\chi^{2}=16.3, p<0.001$ for $\left.w B(P U)\right\}$. However, there was no significant difference in the lifespan of the two single infected strains $w A(P U)$ and lifespans. But what is unambiguous from these results is that the uninfected strain lived the

245 longest, irrespective of the sex of the hosts, indicating that the presence of Wolbachia is associated with the reduction in lifespan and is thus, costly for $N$. vitripennis to maintain. 
$N$. vitripennis, we enumerated the number of copulations an individual male can perform across the infection types. As Fig. 2, indicates, there is indeed a reduction in the capacity of infected males to mate. When compared with the uninfected strain $\mathrm{O}(\mathrm{PU})$, this reduction was most pronounced in $w \mathrm{~B}(\mathrm{PU})(\mathrm{MWU}, \mathrm{U}=30, \mathrm{p}<0.01$ ), followed by $w \mathrm{~A} w \mathrm{~B}(\mathrm{PU})$ and $w \mathrm{~A}(\mathrm{PU})$ which showed similar copulation capacities (MWU: $U=11, p=0.49$ ). The uninfected $0(P U)$ strain produced males with the highest number of copulations $\{\mathrm{MWU}: \mathrm{U}=32, \mathrm{p}<0.05$ for $w \mathrm{~A}(\mathrm{PU})$ and $\mathrm{U}=27, \mathrm{p}<0.05$ for $w \mathrm{~A} w \mathrm{~B}(\mathrm{PU})\}$. Thus, the presence of Wolbachia substantially reduced the number of copulations that a male could perform. However, complex phenotypes like copulation can also be affected by the host genotype. Although all these four strains were derived from the same field-collected isofemale line, continuous culturing in the laboratory can fix specific alleles within them resulting in interstrain divergence. Moreover, it is also known that in Nasonia the effect of Wolbachia-induced phenotype is influenced by the hosts' genetic background (Raychoudhury et al., 2012). Therefore, we cured all these infections again and tested whether the host genotype, rather than Wolbachia, is causing this reduction in the number of copulations by comparing these newly cured strains back with the previously used uninfected strain, 0 (PU). As Fig. 2, indicates, males from most of these re-cured strains showed a marked and significant increase in the number of copulations performed. This number in the re-cured double infected strain, $0(w A w B P U)$, increased to similar levels as shown by $0(P U)$ (an increase of $29 \%, M W U: U=9.5, p=0.2$ ), while also showing a significant increase from its infected counterpart $w A w B(P U)$ (from $73.5 \pm 10.5$ to $94.8 \pm 15.39$, MWU: $U=3, p<0.05$ ). Similarly, the number of copulations for the re-cured single A supergroup infected strain, 0 (wA PU), also increased to the levels of the uninfected strain O(PU) (an increase of 7\%, MWU: $U=20$, 
$272 p=0.76$ ). However, this increase (from $77.5 \pm 6.3$ to $83.5 \pm 12.9$ ) with its infected counterpart

273 was not significant ( $M W U$ : $U=23, p=0.48$ ). The re-cured single $B$ supergroup infection, $O(w B$

274 PU), was the only strain which did not revert to uninfected levels (MWU: $U=22, p<0.05$ )

275 despite showing an increase (from $62.8 \pm 6.6$ to $78.2 \pm 5.1$; MWU: $U=1, p<0.05$ ). However,

276 what is evident is that the presence of Wolbachia is also associated with a reduction in the

277 capability of a male to mate. Furthermore, by curing the infected strains again, we showed that

278 this decrease is not due to the host genotype but is an effect of Wolbachia present in these

279 strains.

Wolbachia infected males deplete their sperm reserves faster than the uninfected ones

281

282

283

284

285

286

287

288

289

290

291

292

N. vitripennis males are prospermatogenic (Boivin et al., 2005), where each male emerges with their full complement of mature sperm and have not been reported to produce any more during the rest of their lifespan (Chirault et al., 2016). Thus, if a single male is mated sequentially with as many females it can mate with, it should eventually run out of this full complement of sperm resulting in an all-male brood even after successful copulation. As Fig. 2, indicates, each male did run out of sperm at the tail end of the continuous mating and produced only male progenies (shown by black dots). We looked at the number of mating done by these males before sperm depletion to see if Wolbachia affects the sperm production in the males. As shown in Fig. 3, the average number of daughter progenies reduced with the number of mating (shown by the primary $Y$-axis on the left), indicating sperm depletion. Similar to copulation numbers, $w \mathrm{~B}(\mathrm{PU})$ males were also the quickest to deplete their sperm reserve \{MWU: $U=27.5, p<0.05$ for $w A(P U)$ and $U=30, p<0.01$ compared for $0(P U)\}$. This was followed 
by $w A w B(P U)$ and $w A(P U)(M W U: ~ U=13, p=0.7)$. However, the uninfected males from $0(P U)$

$48.14 \pm 4.94$ to $50.57 \pm 9.41$ ) was not significantly different from their infected counterpart $w A(P U)$ (MWU: $U=16, p=0.8$ ). Rates of depletion for $O(w A w B P U)$ also increased up to the levels of $O(P U)$ (an increase of 15.2\%, MWU: $U=21, p=0.66$ ). Again, the cured strain $O(w B P U)$, increased from $w \mathrm{~B}(\mathrm{PU})$ (from $41 \pm 1.67$ to $47.6 \pm 6.0, \mathrm{MWU}$ : $\mathrm{U}=0, \mathrm{p}<0.05$ ) but was still lower in number than $0(P U)$ (MWU: $U=23, p<0.05$ ). These results indicate that the presence of Wolbachia has a significant negative impact on the number of sperm produced or utilized by the infected strains.

\section{Wolbachia infected females produce fewer offspring}

Wolbachia is known to have a negative impact on the progeny family size of its host (Hoffmann infection strains and their recently cured counterparts.

312 broods produced by the virgin females of the Wolbachia infected strains. When compared with

313 the uninfected strain $\mathrm{O}(\mathrm{PU})$, this reduction was most pronounced in wAwB(PU) (MWU: 
$\mathrm{U}=21151.5, \mathrm{p}<0.01$ ) followed by $w \mathrm{~B}(\mathrm{PU})(\mathrm{MWU}: \mathrm{U}=19880.5, \mathrm{p}<0.05) . w \mathrm{~B}(\mathrm{PU})$ and $w \mathrm{~A} w \mathrm{~B}(\mathrm{PU})$ family size when compared with O(PU) (MWU: $\mathrm{U}=17191, \mathrm{p}=0.39$ ) and $w \mathrm{~B}(\mathrm{PU})$ (MWU:

$317 \mathrm{U}=17284, \mathrm{p}=0.26$ ) but had larger all-male broods sizes than $w A w B(P U)$ (MWU: $U=18252, p<$ 0.05). We also compared the recently cured single and double infected strains with the infected parental strains. The recently cured strains $\mathrm{O}(w \mathrm{~B} \mathrm{PU})$ and $\mathrm{O}(w \mathrm{~A} w \mathrm{~B} \mathrm{PU})$ showed marginal increase in the family size which was comparable to the uninfected strain O(PU) \{an increase of 1.5\%, MWU: $U=11554, p=0.29$ for $0(w B P U)$; an increase of $2 \%, M W U: U=10798, p=0.21$ for $\mathrm{O}(w \mathrm{~A} w \mathrm{~B} \mathrm{PU})$ \}. However this marginal increase ffrom $29.84 \pm 12.94$ to $31.44 \pm 10.30$ for $\mathrm{O}(w \mathrm{~B}$ PU) and from $28.99 \pm 11.60$ to $30.97 \pm 11.48$ for $0(w A w B$ PU $)\}$ was not significantly different from their infected counterparts $w B(P U)$ (MWU: $\mathrm{U}=9963.5, \mathrm{p}=0.34$ ) and $w A w B(P U)$ (MWU: 0.22).

Similar to the virgin females, the reduction was also observed for average family sizes of mated females as shown in Fig. 4(B). When compared with the uninfected strain $0(P U)$, this reduction is most pronounced in $w \mathrm{~B}(\mathrm{PU})$ (MWU: 15582, $\mathrm{p}<0.01$ ) and $w A w \mathrm{~B}(\mathrm{PU})$ (MWU: $\mathrm{U}=16303, \mathrm{p}<$ 0.01). However, $w \mathrm{~B}(\mathrm{PU})$ and $w \mathrm{~A} w \mathrm{~B}(\mathrm{PU})$ show similar levels of family sizes (MWU: $13732.5, \mathrm{p}=$ 0.55). Interestingly, wA(PU) strain shows similar levels of family size as $0(P U)$ (MWU: $\mathrm{U}=11396.5, \mathrm{p}=0.86$ ) but had larger family sizes than $w \mathrm{~B}(\mathrm{PU})(\mathrm{MWU}: \mathrm{U}=14080, \mathrm{p}<0.01$ ) and $w A w B(P U)$ (MWU: $\mathrm{U}=14682, \mathrm{p}<0.05$ ). Upon curing, the average family sizes of the recently 
11.8\%, MWU: $U=13295, p=0.61$ ) showing a significant increase from the infected counterpart $w A w B(P U)$ (from $29.49 \pm 10.67, \mathrm{MWU}: \mathrm{U}=12023, \mathrm{p}<0.05)$. The recently cured strain $\mathrm{O}(w \mathrm{AU})$ did not show any significant increase from the infected counterpart wA(PU) (MWU: U=8385.5 p $=0.69$ ) and was comparable to O(PU) (MWU: U=9022.5, $p=0.5$ ). However, the recently cured strain $O(W B P U)$ did not increase to the levels of the uninfected strain $O(P U)$ (an increase of 4.3\%, MWU: $U=16782, p<0.05$ ). The marginal increase in the family size (from $28.72 \pm 10.46$ to $29.97 \pm 8.59)$ was not significantly different from the parent strain $w B(P U)$ (MWU: $U=13854, p=$ 0.47).

To understand whether this difference in the family size of the mated females is due to the production of fewer daughters or sons or both, we compared their numbers separately for the

346 four strains (Fig. 4C). No difference was observed in the number of sons produced by the mated

347 females. However, significant differences were observed in the number of daughters produced.

348 When compared to the uninfected strain $\mathrm{O}(\mathrm{PU}), w \mathrm{~B}(\mathrm{PU})$ and $w \mathrm{~A} w \mathrm{~B}(\mathrm{PU})$ showed the least number of daughters produced \{MWU: 15964, $p<0.01$ for $w B(P U)$ and $U=16283, p<0.01$ for $w A w B(P U)\}$ whereas $w \mathrm{~B}(\mathrm{PU})$ and $w A w B(P U)$ produced nearly equal number of daughters to 0 (PU) (MWU: $\mathrm{U}=11543, \mathrm{p}=0.98$ ) but higher in number than $w \mathrm{~B}(\mathrm{PU})$ and $w A w \mathrm{~B}(\mathrm{PU})\{\mathrm{MWU}$ :

$353 \mathrm{U}=14201, \mathrm{p}<0.01$ for $w \mathrm{~B}(\mathrm{PU})$ and $\mathrm{MWU}: \mathrm{U}=14372, \mathrm{p}<0.05$ for $w A w \mathrm{~B}(\mathrm{PU})\}$. Upon curing, the recently cured $O(w A w B P U)$ reverted to the levels of the uninfected strain $O(P U)$ (MWU: $U=$ $13545, p=0.42$ ) showing a significant increase in the number of daughters from the infected counterpart $w A w B(P U)$ (MWU: $U=12331, p<0.039)$. The recently cured strain $0(w A P U)$ did not show any increase in the number of daughters produced from their infected counterpart 
$w A(P U)$ (MWU: $U=8468 p=0.79$ ) and was also comparable to $0(P U)$ (MWU: $U=9330, p=0.84$ ).

However, recently cured strain $\mathrm{O}(W \mathrm{~B} \mathrm{PU})$ did not increase to the levels of the uninfected strain

O(PU) (MWU: U=16749.5, $p<0.01$ ).

Wolbachia negatively impacts the fecundity of infected females

362

363

$\mathrm{U}=2388, \mathrm{p}<0.05$ ) and was similar to the uninfected strain $0(P U)$ (MWU: $U=3799.5, p=0.75$ ) .

To check whether the differences in the family sizes between the different infection strains are due to the number of eggs being laid by the females, we looked at the fecundity of both virgin and mated females across the strains. Among the virgin females (Fig. 5A), wAwB(PU) had the least fecundity (MWU: $\mathrm{U}=5042, \mathrm{p}<0.01$ ) when compared to the uninfected strain $\mathrm{O}(\mathrm{PU}$ ). However, there was no significant difference between $0(P U)$ and $w A(P U)(M W U: U=2632.5, p=$ 0.34 ) and also between $0(\mathrm{PU})$ and $w \mathrm{~B}(\mathrm{PU})$ (MWU: $\mathrm{U}=2052.5, \mathrm{p}=0.71$ ).

Among the mated females (Fig. 5B), wAwB(PU) again had the least fecundity (MWU: $\mathrm{U}=2461.5$, $\mathrm{p}<0.01$ ) when compared to the uninfected strain $\mathrm{O}(\mathrm{PU})$. This was followed by $w \mathrm{~B}(\mathrm{PU})$ having similar fecundity as that of $w A w B(P U)$ (MWU: $U=1479.5, p=0.24$ ) and $w A(P U)$ (MWU: $\mathrm{U}=2822.5, \mathrm{p}=0.22)$. However, $w \mathrm{~A}(\mathrm{PU})$ showed higher fecundity than $w A w \mathrm{~B}(\mathrm{PU})(\mathrm{MWU}$ : The double infected strain showed the least fecundity, both for the virgin and mated females, suggesting an effect of Wolbachia on egg production in females. The assay also established that the difference in family sizes is due to the differences in the fecundity of the females.

\section{Relative Wolbachia density in single and multiple Wolbachia infection strains}


377 Wolbachia density has a major role to play in expressing the effects of the infection on host

378 biology (Hoffmann et al., 1996; Min \& Benzer, 1997). An increase in cellular Wolbachia density

379 is often associated with a greater expression of their effects (Breeuwer \& Werren, 1993). Thus,

380 we estimated Wolbachia titers across different developmental stages of $N$. vitripennis males

381 and females. In the case of males (Fig. 6A) wA(PU) had the lowest Wolbachia density across

382 different larval and pupal developmental stages when compared with $w B(P U)$ (MWU: $U=11, p<$

3830.01 ) and $w A w B(P U)(M W U: ~ U=12, p<0.01$ ) This difference was more prominent at the adult

384 stage. However, no such differences were found between $w \mathrm{~B}(\mathrm{PU})$ and $w A w \mathrm{~B}(\mathrm{PU})(\mathrm{MWU}: \mathrm{U}=51$, $385 p=0.56)$.

386 In the case of females (Fig. 6B), wA(PU) showed lower levels of Wolbachia when compared to $387 w A w B(P U)$ (MWU: $\mathrm{U}=8, \mathrm{p}<0.05$ ) again at the late pupal and adult stage. However, no 388 difference was observed between $w \mathrm{~A}(\mathrm{PU})$ and $w \mathrm{~B}(\mathrm{PU})(\mathrm{MWU}: \mathrm{U}=12, \mathrm{p}=0.12$ ) and also $w \mathrm{~B}(\mathrm{PU})$ 389 and $w A w B(P U)$ (MWU: $U=19, p=0.5$ ).

\section{Discussion}

$391 \mathrm{Cl}$-inducing Wolbachia are known to have negative effects on various physiological traits in the

392 vast majority of its host population (summarized in Table 1). The present study also suggests 393 such effects, or a "cost", associated with the maintenance of Wolbachia infection in $N$. 394 vitripennis. This is in contrast to the previous reports suggesting positive fitness effects (Stolk C 395 \& Stouthamer R, 1996) and no fitness effects (Bordenstein \& Werren, 2000) of Wolbachia on N. 396 vitripennis. However, several unique conclusions emerge from the study. 
397 Our results (summarized in Table 2) demonstrate a sex-independent cost of the presence of 398 single and multiple Wolbachia infections. Many phenotypes show reduction across the sexes 399 such as longevity (Fig. 1) where infected males and females showed reduced lifespan. When 400 compared with the uninfected strain $\mathrm{O}(\mathrm{PU})$, the Wolbachia infected strains $w \mathrm{~B}(\mathrm{PU})$ and 401 $w A w B(P U)$ have reduced life spans. However, sex-specific variations were also observed among 402 the infected strains where wA(PU), in females, had the shortest life span, while in the case of 403 males, had a greater life span than $w A w B(P U)$.

404 Wolbachia affected the reproductive capabilities of the infected males and reduced their 405 copulation capability (Fig. 2) and also led to quicker sperm depletion (Fig. 3). Such negative 406 effects on reproductive traits were also observed in females where the infected females 407 produced fewer progenies (Fig. 4) compared to O(PU). These differences were elicited at the 408 level of female fecundity where the number of eggs being laid by the infected females was 409 fewer (Fig. 5) indicating that the negative effects of Wolbachia manifested themselves even 410 before the egg-laying stage. However, the egg to larval to pupal stage mortality could also have 411 an effect on the brood sizes but these were not assayed.

412 In most cases, these negative effects disappeared with the removal of Wolbachia, indicating the 413 role of Wolbachia in producing these negative effects and not the host genotype. In phenotypes 414 like family sizes, the recently cured strains showed a significant increase suggesting that the 415 negative effects are due to the presence of Wolbachia. However, 0(wB PU) did not revert to the 416 levels of $\mathrm{O}(\mathrm{PU})$ in the number of copulations performed and sperm depletion assays. A possible 417 reason can be some residual effects of the parent genotype in $0(w B P U)$ males. 
418 Our results also demonstrate supergroup-specific negative effects on the host. Supergroup B

419 Wolbachia is costlier to maintain in both the sexes than supergroup $\mathrm{A}$. While $w \mathrm{~B}(\mathrm{PU})$ shows

420 strong effects on all the traits studied across the sexes, wA(PU) had significant negative effects

421 only on the reproductive traits of the males and the longevity of females. $w A(P U)$ females, as an

422 exception, had their family sizes comparable to O(PU). These observations are unique as no

423 comprehensive data is available on the supergroup-specific cost of Wolbachia infections.

424 The higher cost of maintenance of supergroup B Wolbachia can be an attribute of the $\mathrm{Cl}$

425 phenotype induced by supergroup B Wolbachia. Complete $\mathrm{Cl}$ ( i.e, nearly $100 \% \mathrm{Cl}$ ) are rare

426 events reported mainly for supergroup B Wolbachia in Culex pipiens, Aedes aegypti (Sinkins et

427 al., 2005; Xi et al., 2005), and in N. vitripennis as well (supplementary Fig. 1 and Bordenstein et

428 al., 2006a). This essentially means that nearly the entire sperm complement of each male has

429 the Wolbachia-induced $\mathrm{Cl}$ modification and correspondingly, nearly all the eggs from the

430 females have the rescue effect (Werren et al., 2008). Introducing $100 \%$ modification and rescue

431 would necessitate relatively high Wolbachia titers to be maintained in both sexes which in turn

432 can cause an elevated nutritional burden, eventually resulting in negative effects on the

433 physiological traits of the host.

434 Previous reports have suggested a direct correlation between Wolbachia density and the level

435 of $\mathrm{Cl}$ (Breeuwer \& Werren, 1993; Noda et al., 2001; Ikeda et al., 2003; Dutton \& Sinkins, 2004;

436 Ruang-Areerate \& Kittayapong, 2006). Our results also suggest that the cost of Wolbachia

437 maintenance is correlated with the density of Wolbachia strains present in the host. Wolbachia

438 density in both the sexes differs in the late pupal stages (Day 11, Day 12) and adult stages. Thus, 
in the case of females, $w A w B(P U)$, which shows a high bacterial load, has reduced fecundity and longevity. Similarly, in the case of males, the $w A w B(P U)$ strain shows a reduced number of copulations and the number of sperm produced/transferred. $w \mathrm{~B}(\mathrm{PU})$, therefore causes substantial detrimental effects on both the sexes of the host which is similar to the levels found in $w A w B(P U)$. This again can be explained by the high Wolbachia load in both sexes. In contrast, $w A(P U)$ showed minimal effect on the female hosts, with the longevity of females being an exception. A possible explanation for this can be the relatively low density of $w \mathrm{~A}(\mathrm{PU})$ across the different developmental stages (Fig. 6) as compared to the other infections. Moreover, supergroup A infected strains are known to have relatively higher levels of phage density (Bordenstein et al., 2006). According to this phage density model, higher phage density has an impact on the level of $\mathrm{Cl}$ caused by supergroup A strains, where it leads to a significant reduction in Wolbachia titer and hence has a reduction effect on $\mathrm{Cl}$ phenotype. Our results also conform with the previous reports of the positive correlation between Wolbachia abundance and the level of $\mathrm{Cl}$ induced not only in $\mathrm{N}$. vitripennis (Bordenstein et al., 2006b) but in other insect taxa as well (Ijichi et al., 2002; Kondo et al., 2002). wB(PU) Wolbachia showed complete $\mathrm{Cl}$ and had higher Wolbachia titers in both males and females, which was also comparable to that of the $w A w B(P U)$, whereas $w \mathrm{~A}(\mathrm{PU})$ had the lowest Wolbachia titers among the three strains and showed incomplete $\mathrm{Cl}$ (supplementary Fig. 1). Thus, higher levels of $w \mathrm{~B}(\mathrm{PU})$ than $w \mathrm{~A}(\mathrm{PU})$ also explain the more severe effects of $w \mathrm{~B}(\mathrm{PU})$ than $w \mathrm{~A}(\mathrm{PU})$.

The low levels of $w \mathrm{~A}(\mathrm{PU})$ and the associated negative effects on host physiology raise questions on the maintenance of $w \mathrm{~A}(\mathrm{PU})$ infection in $N$. vitripennis. Since supergroup $\mathrm{B}$ appears to be a "stronger" Wolbachia than supergroup A, any competition for nutritional resources and niche 
461 habitation between them should drive out supergroup A Wolbachia from the hosts. But wA(PU)

462 is observed to have less severe effects on the females with longevity being the only factor to

463 have any negative effects. Supergroup A Wolbachia in N. vitripennis is closely related to other A

464 supergroup Wolbachia like wMel in $D$. melanogaster and $w \mathrm{Ha}, w \mathrm{Au}$, and $w \mathrm{Ri}$ in $D$. simulans

465 (Díaz-Nieto et al., 2021). The presence of supergroup A Wolbachia in N. vitripennis could be due

466 to such defenses against viral infections (Teixeira et al., 2008; Bhattacharya et al., 2017;

467 Pimentel et al., 2021) but remain untested.

468 The varied effects of Wolbachia can be dependent on host strain as well as on a particular

469 Wolbachia supergroup. Our data suggest that supergroup B Wolbachia behaves more like a

470 parasite in both the sexes while supergroup A Wolbachia had detrimental effects mostly in the

471 case of males. This is contrary to what is expected as the two Wolbachia supergroups being

472 maternally transmitted, should affect the males more in comparison to the females as males

473 are a dead-end for Wolbachia transmission.

474 Our experiments indicate an additive or synergistic effect of the presence of the two different

475 Wolbachia supergroups in the double infected strain wAwB(PU). Evidence of such effects can

476 be seen in traits like male longevity (additive effect) where the deficit in longevity for

$477 w \mathrm{~A} w \mathrm{~B}(\mathrm{PU})$ is equal to the total deficits caused by $w \mathrm{~A}(\mathrm{PU})$ and $w \mathrm{~B}(\mathrm{PU})$. Similarly, for traits like

478 female longevity, virgin female family size, and female fecundity, the negative effects on strain

$479 w \mathrm{~A} w \mathrm{~B}(\mathrm{PU})$ appear to be a combined effect of $w \mathrm{~A}(\mathrm{PU})$ and $w \mathrm{~B}(\mathrm{PU})$ (synergistic effect). Since the

480 two supergroup infections are bidirectionally incompatible with each other, it is plausible that 
481 they are also competing for the host nutrition, which can further enhance the negative impacts

482 of these infections.

483 The negative fitness effects of $\mathrm{Cl}$-inducing Wolbachia, and nutritional competition raises 484 important questions on the maintenance of these endosymbionts over long evolutionary time 485 scales. Theoretical studies predict that for the long-term persistence of these maternally 486 inherited reproductive parasites, they can eventually evolve towards mutualism (Prout, 1994; 487 Turelli, 1994). Various theories on endosymbiosis also support the vertical transmission of 488 endosymbionts, as the spread of the symbiont depends on the reproductive success of the host 489 itself (Anderson \& May 1982; Ewald, 1987; Bull et al., 1991; Lipsitch et al., 1995). Hence, 490 mutualism can be a preferred way for endosymbionts to ensure their persistence. Moreover, if 491 there are indeed some adverse effects of maintaining Wolbachia, then hosts would be under 492 strong selection pressure to develop immunity against them. Evidence suggests that there are 493 examples of such emergence of host genetic factors upon Wolbachia infection in hosts 494 Drosophila and mosquitoes (Zug \& Hammerstein, 2015). Host suppressor alleles have been 495 identified conferring resistance against feminizing (Rigaud et al., 1999) and male-killing 496 Wolbachia (Hornett et al., 2006). However, no such host genetic factors have been found for $\mathrm{Cl}-$ 497 inducing Wolbachia, especially in N. vitripennis. A possible explanation for the maintenance of 498 these multiple infections comes from the high efficiency of transmission of these infections in 499 N. vitripennis, where these infections also show nearly $100 \%$ transmission as well as $\mathrm{Cl}$ 500 (Breeuwer and Werren, 1990). For many Wolbachia-arthropod associations, the pathogenicity 501 and transmission efficiency is mediated by bacterial titers (Correa \& Ballard, 2016). Our results 502 also show higher Wolbachia density for double infected strain, which can facilitate its 
503 transmission and maintenance in the host. Another possibility can be that these Wolbachia

504 infections in N. vitripennis are relatively recent, the evidence of which comes from the rapid

505 spread of Wolbachia in populations of N. vitripennis across North America and Europe

506 (Raychoudhury et al., 2010b). These recent infections, although bearing a cost on the host at

507 present, might eventually lead to the host immunity against the infection.

\section{Acknowledgments}

509 We thank the Indian Institute of Science Education and Research (IISER) Mohali for the funding 510 and graduate fellowship for AT. Partial funding was obtained from Grant No.

511 BT/PR14278/BRB/10/1417/2015, Department of Biotechnology, Government of India, awarded

512 to Nagaraj Guru Prasad (Associate Professor, Biological Sciences, IISER Mohali) and RR.

\section{Competing Interests}

514 The authors declare no conflict of interest.

\section{Author Contribution}

516 AT conceived the idea and planned the study. AT performed the experiments. AT collected the

517 data. RB performed the pilot experiments for female fecundity. AT analyzed the data. AT, RS,

518 and RR were involved in data representation and statistical analysis. AT and RR wrote the 519 manuscript.

\section{Data Archiving}


521 The raw data for all the experiments have been archived at Dryad with DOI:

$52210.5061 /$ dryad.w0vt4b8s9

523 URL: https://datadryad.org/stash/share/gKvBkEcYjmrNULX4F53Ktu5Pt-P1pcXBnbA5Ib_HEPo

524

525 References

526 Anderson RM, May RM (1982). Coevolution of hosts and parasites, Parasitology, 85, 411-42

527 https://doi.org/10.1017/S0031182000055360

528 Bandi C, Anderson TJC, Genchi C, Blaxter ML (1998). Phylogeny of Wolbachia in filarial

529 nematodes. Proc. R. Soc. Lond. B, 265, 2407-2413

530 https://dx.doi.org/10.1098\%2Frspb.1998.0591

531 Bhattacharya T, Newton ILG, Hardy RW (2017). Wolbachia elevates host methyltransferase

532 expression to block an RNA virus early during infection. PLoS Pathog, 13

533 https://doi.org/10.1371/journal.ppat.1006427

534 Boivin G, Jacob S, Damiens D (2005). Spermatogeny as a life-history index in parasitoid wasps.

$535 \quad$ Oecologia, 143, 198-202.

536 https://www.jstor.org/stable/20062237

537 Bordenstein SR, Bordenstein SR (2011). Temperature affects the tripartite interactions between

538 bacteriophage WO, Wolbachia, and cytoplasmic incompatibility. PLoS One, 6

539 https://doi.org/10.1371/journal.pone.0029106

540 Bordenstein SR, Marshall ML, Fry AJ, Kim U, Wernegreen JJ (2006a). The tripartite associations

541 between bacteriophage, Wolbachia, and arthropods. PLoS Pathogen, 2, 384-393. 
542 https://doi.org/10.1371/journal.ppat.0020043

543 Bordenstein SR, Marshall ML, Fry AJ, Kim U, Wernegreen JJ (2006b). The tripartite associations

544 between bacteriophage, Wolbachia, and arthropods. PLoS Pathogen, 2, 384-393.

545 https://doi.org/10.1371/journal.ppat.0020043

546 Bordenstein SR, Werren JH (2000). Do Wolbachia influence fecundity in Nasonia vitripennis?

$547 \quad$ Heredity (Edinb), 84, 54-62.

548 https://doi.org/10.1046/j.1365-2540.2000.00637.x

549 Breeuwer JAJ, Werren JH (1990). Microorganisms associated with chromosome destruction and 550 reproductive isolation between two insect species. Nature, $346,558-560$.

551 https://doi.org/10.1038/346558a0

552 Breeuwer JAJ, Werren JH (1993). Cytoplasmic Incompatibility and Bacterial Density in Nasonia 553 vitripennis. Genetics ,135(2), 565-574.

554 https://www.ncbi.nlm.nih.gov/pmc/articles/PMC1205656/

555 Bull JJ, Molineux IJ, Rice WR (1991). Selection of benevolence in a host-parasite system.

$556 \quad$ Evolution (N Y), 45, 875-882.

557 https://doi.org/10.1111/j.1558-5646.1991.tb04356.x

558 Caragata EP, Rancès E, O’Neill SL, McGraw EA (2014). Competition for Amino Acids Between

559 Wolbachia and the Mosquito Host, Aedes aegypti. Microb Ecol, 67, 205-218.

560

https://doi.org/10.1007/s00248-013-0339-4

561 Caspari E, Watson GS (1959). On the Evolutionary Importance of Cytoplasmic Sterility in Mosquitoes, JSTOR, 13, 568-570. 
564

565

566

567

568

569

570

571

572

573

574

575

576

577

578

579

580

581

582

583

584

585

Chirault M, van de Zande L, Hidalgo K, Chevrier C, Bressac C, Lécureuil C (2016). The spatiotemporal partitioning of sperm by males of the prospermatogenic parasitoid Nasonia vitripennis is in line with its gregarious lifestyle. J Insect Physiol, 91-92, 10-17.

https://doi.org/10.1016/j.jinsphys.2016.06.002

Correa CC, Ballard JWO (2016). Wolbachia Associations with Insects: Winning or Losing Against a Master Manipulator. Front Ecol Evol, 3

https://doi.org/10.3389/fevo.2015.00153

D JF and AJ V (1996). A Causal Ethological Analysis of the Courtship Behaviour of an Insect ( The Parasitic Wasp Nasonia vitripennis , Hym ., Pteromalidae ). Behaviour, 133, 1051-1075.

https://www.jstor.org/stable/4535410

Dedeine F, Vavre F, Fleury F, Loppin B, Hochberg ME, Boulétreau M (2001). Removing symbiotic Wolbachia bacteria specifically inhibits oogenesis in a parasitic wasp. Proc Natl Acad Sci U $S A, 98,6247-6252$.

https://doi.org/10.1073/pnas.101304298

Díaz-Nieto LM, Gil MF, Lazarte JN, Perotti MA, Berón CM (2021). Culex quinquefasciatus carrying Wolbachia is less susceptible to entomopathogenic bacteria. Sci Rep, 11, 1-9.

https://doi.org/10.1038/s41598-020-80034-5

Dobson SL, Rattanadechakul W, Marsland EJ (2004). Fitness advantage and cytoplasmic incompatibility in Wolbachia single- and superinfected Aedes albopictus. Heredity (Edinb), 93, 135-142.

https://doi.org/10.1038/sj.hdy.6800458

Dowling DK, Simmons LW (2009). Reactive oxygen species as universal constraints in life-history 
evolution. Proc R Soc B Biol Sci, 276, 1737-1745.

587

588

589

590

591

592

593

594

595

596

597

598

599

600

601

602

603

604

605

606

607

https://doi.org/10.1098/rspb.2008.1791

Duron O, Labbé P, Berticat C, Rousset F, Guillot S, Raymond M, et al. (2006). High Wolbachia Density Correlates With Cost of Infection for Insecticide Resistant Culex Pipiens

Mosquitoes. Evolution ( $N Y), 60,303$.

https://pubmed.ncbi.nlm.nih.gov/16610322/

Dutton TJ, Sinkins SP (2004). Strain-specific quantification of Wolbachia density in Aedes

albopictus and effects of larval rearing conditions. Insect Mol Biol, 13, 317-322.

https://doi.org/10.1111/j.0962-1075.2004.00490.x

Ewald PW (1987). Transmission Modes and Evolution of the Parasitism-Mutualism Continuum. Ann N Y Acad Sci, 503, 295-306.

https://doi.org/10.1111/j.1749-6632.1987.tb40616.x

Fleury F, Vavre F, Ris N, Fouillet $P$, Boulétreau M (2000). Physiological cost induced by the maternally-transmitted endosymbiont Wolbachia in the Drosophila parasitoid Leptopilina heterotoma. Parasitology, 121, 493-500.

https://doi.org/10.1017/s0031182099006599

Foster J, Ganatra M, Kamal I, Ware J, Makarova K, Ivanova N, et al. (2005). The Wolbachia genome of Brugia malayi: Endosymbiont evolution within a human pathogenic nematode (NA Moran, Ed.). PLoS Biol, 3, 0599-0614.

https://doi.org/10.1371/journal.pbio.0030121

Fry AJ, Palmer MR, Rand DM (2004). Variable fitness effects of Wolbachia infection in Drosophila melanogaster. Heredity (Edinb), 93, 379-389. 
608

609

610

611

612

613

614

615

616

617

618

619

620

621

622

623

624

625

626

627

628

629

https://doi.org/10.1038/sj.hdy.6800514

Hertig M and Wolbach S B (1924). Studies on Rickettsia-like micro-organisms in insects. JMed

Res, 329-374.7.

https://www.ncbi.nlm.nih.gov/pmc/articles/PMC2041761/

Hoffmann AA, Clancy D, Duncan J (1996). Naturally-occurring Wolbachia infection in Drosophila simulans that does not cause cytoplasmic incompatibility. Heredity (Edinb), 76, 1-8.

https://doi.org/10.1038/hdy.1996.1

Hohmann CL, Luck RF, Stouthamer R (2001). Effect of Wolbachia on the Survival and Reproduction of Trichogramma kaykai Pinto \& Stouthamer (Hymenoptera:

Trichogrammatidae). Neotropical Entomology, 30(4), 607-612

https://doi.org/10.1590/S1519-566X2001000400015

Hornett EA, Charlat S, Duplouy AMR, Davies N, Roderick GK, Wedell N, et al. (2006). Evolution of male-killer suppression in a natural population. PLOS Biol, 4, 1643-1648.

https://doi.org/10.1371/journal.pbio.0040283

ljichi N, Kondo N, Matsumoto R, Shimada M, Ishikawa H, Fukatsu T (2002). Internal spatiotemporal population dynamics of infection with three Wolbachia strains in the adzuki bean beetle, Callosobruchus chinensis (Coleoptera: Bruchidae). Appl Environ Microbiol, 68, 4074-4080.

https://dx.doi.org/10.1128\%2FAEM.68.8.4074-4080.2002

Ikeda T, Ishikawa H, Sasaki T (2003). Infection density of Wolbachia and level of cytoplasmic incompatibility in the Mediterranean flour moth, Ephestia kuehniella. J Invertebr Pathol, $84,1-5$. 
630

631

632

633

634

635

636

637

638

639

640

641

642

643

644

645

646

647

648

649

650

651

https://doi.org/10.1016/s0022-2011(03)00106-x

Kobayashi DY, Crouch JA (2009). Bacterial/Fungal interactions: from pathogenes to mutualistic endosymbionts. Annu Rev Phytopathol, 47, 63-82.

https://doi.org/10.1146/annurev-phyto-080508-081729

Kondo N, Ijichi N, Shimada M, Fukatsu T (2002). Prevailing triple infection with Wolbachia in Callosobruchus chinensis (Coleoptera: Bruchidae). Mol Ecol, 11, 167-180.

https://doi.org/10.1046/j.0962-1083.2001.01432.x

Landmann F (2019). The Wolbachia Endosymbionts. Microb Spectrum, Am Soc Microbiol, 7, $139-153$.

https://doi.org/10.1128/microbiolspec.bai-0018-2019

Lipsitch M, Nowak MA, Ebert D, May RM (1995). The population dynamics of vertically and horizontally transmitted parasites, Proc. R. Soc. Lond. B, 260, 321-327

https://doi.org/10.1098/rspb.1995.0099

McMeniman CJ, Lane R V., Cass BN, Fong AWC, Sidhu M, Wang YF, et al. (2009). Stable introduction of a life-shortening Wolbachia infection into the mosquito Aedes aegypti. Science, 323, 141-144.

https://doi.org/10.1126/science.1165326

Miller WJ, Ehrman L, Schneider D (2010). Infectious speciation revisited: Impact of symbiontdepletion on female fitness and mating behavior of Drosophila paulistorum. PLoS Pathog 6.

https://doi.org/10.1371/journal.ppat.1001214

Min KT, Benzer S (1997). Wolbachia, normally a symbiont of Drosophila, can be virulent, causing 
degeneration and early death. Proc Natl Acad Sci U S A, 94, 10792-10796.

653

654

655

656

657

658

659

660

661

662

663

664

665

666

667

668

669

670

671

672

673

https://doi.org/10.1073/pnas.94.20.10792

Monaghan P, Metcalfe NB, Torres R (2009). Oxidative stress as a mediator of life history tradeoffs: Mechanisms, measurements and interpretation. Ecol Lett, 12, 75-92.

https://doi.org/10.1111/j.1461-0248.2008.01258.x

Moné Y, Monnin D, Kremer N (2014). The oxidative environment: A mediator of interspecies communication that drives symbiosis evolution. Proc R Soc B Biol Sci, 281.

https://dx.doi.org/10.1098\%2Frspb.2013.3112

Noda H, Koizumi Y, Zhang Q, Deng K (2001). Infection density of Wolbachia and incompatibility level in two planthopper species, Laodelphax striatellus and Sogatella furcifera. Insect Biochem Mol Biol, 31, 727-737.

https://doi.org/10.1016/s0965-1748(00)00180-6

Olsen K, Reynolds KT, Hoffmann AA (2001). A field cage test of the effects of the endosymbiont Wolbachia on Drosophila melanogaster. Heredity (Edinb), 86, 731-737.

https://doi.org/10.1046/j.1365-2540.2001.00892.x

Pan X, Pike A, Joshi D, Bian G, McFadden MJ, Lu P, et al. (2018). The bacterium Wolbachia exploits host innate immunity to establish a symbiotic relationship with the dengue vector mosquito Aedes aegypti. ISME J, 12, 277-288.

https://doi.org/10.1038/ismej.2017.174

Pan X, Zhou G, Wu J, Bian G, Lu P, Raikhel AS, et al. (2012). Wolbachia induces reactive oxygen species (ROS)-dependent activation of the Toll pathway to control dengue virus in the mosquito Aedes aegypti. Proc Natl Acad Sci U S A, 109. 
674

675

676

677

678

679

680

681

682

683

684

685

686

687

688

689

690

691

692

693

694

695

https://doi.org/10.1073/pnas.1116932108

Perrot-Minnot M-JJ, Guo LR, Werren JH (1996). Double Infections with Wolbachia in the

Parasitic Wasp Nasonia vitripennis: Effects on Compatibility. Genetics, 143, 961-972.

https://doi.org/10.1093/genetics/143.2.961

Pike N, Kingcombe R (2009). Antibiotic treatment leads to the elimination of Wolbachia

endosymbionts and sterility in the diplodiploid collembolan Folsomia candida. BMC Biol, 7,

54.

https://bmcbiol.biomedcentral.com/articles/10.1186/1741-7007-7-54\#citeas

Pimentel AC, Cesar CS, Martins M, Cogni R (2021). The Antiviral Effects of the Symbiont Bacteria Wolbachia in Insects. Front Immunol, 11, 1-10.

https://doi.org/10.3389/fimmu.2020.626329

Poinsot D, Merçot H (1997). Wolbachia infection in Drosophila simulans: Does the female host bear a physiological cost? Evolution ( $N Y), 51,180-186$.

https://doi.org/10.1111/j.1558-5646.1997.tb02399.x

Ponton F, Wilson K, Holmes A, Raubenheimer D, Robinson KL, Simpson SJ (2014).

Macronutrients mediate the functional relationship between Drosophila and Wolbachia.

Proc R Soc B Biol Sci, 282.

https://doi.org/10.1098/rspb.2014.2029

Prout T (1994). Some evolutionary possibilities for a microbe that causes incompatibility in its host. Evolution (NY), 48, 909-911.

https://doi.org/10.2307/2410244

Raychoudhury R, Grillenberger BK, Gadau J, Bijlsma R, Van De Zande L, Werren JH, et al. (2010). 
Phylogeography of Nasonia vitripennis (Hymenoptera) indicates a mitochondrial-

Wolbachia sweep in North America. Heredity (Edinb), 104, 318-326.

https://doi.org/10.1038/hdy.2009.160

Rigaud T, Moreau J, Juchault P (1999). Wolbachia infection in the terrestrial isopod Oniscus asellus: Sex ratio distortion and effect on fecundity. Heredity (Edinb), 83, 469-475.

https://www.nature.com/articles/6885990\#citeas

Rousset F, Bouchon D, Pintureau B, Juchault P, Solignac M (1992). Wolbachia endosymbionts responsible for various alterations of sexuality in arthropods. Proc $R$ Soc B Biol Sci, 250, 9198.

https://doi.org/10.1098/rspb.1992.0135

Ruang-Areerate T, Kittayapong P (2006). Wolbachia transinfection in Aedes aegypti: A potential gene driver of dengue vectors. Proc Natl Acad Sci U S A, 103, 12534-12539.

https://dx.doi.org/10.1073\%2Fpnas.0508879103

Selman C, Blount JD, Nussey DH, Speakman JR (2012). Oxidative damage, ageing, and lifehistory evolution: Where now? Trends Ecol Evol, 27, 570-577.

https://doi.org/10.1016/j.tree.2012.06.006

Sinkins SP, Walker T, Lynd AR, Steven AR, Makepeace BL, Godfray HCJ, et al. (2005). Wolbachia variability and host effects on crossing type in Culex mosquitoes. Nature, 436, 257-260.

https://doi.org/10.1038/nature03629

Slatko BE, Taylor MJ, Foster JM (2010). The Wolbachia endosymbiont as an anti-filarial nematode target. Symbiosis, 51, 55-65.

https://doi.org/10.1007/s13199-010-0067-1 
718 Stolk C and Stouthamer R (1996). Influence of a Cytoplasmic Incompatibility-Inducing Entomol NEV Amsterdam, 7.

https://research.wur.nl/en/publications/influence-of-a-cytoplasmic-incompatibility-inducing-

\section{3}

724

725

726

727

728

729

730

731

732

733

734

735

736

737

738

739

wolbachia-on-

Sumida Y, Katsuki M, Okada K, Okayama K, Lewis Z (2017). Wolbachia induces costs to lifehistory and reproductive traits in the moth, Ephestia kuehniella. J Stored Prod Res, 71, 93-

98.

10.1016/j.jspr.2017.02.003

Tagami Y, Miura K, Stouthamer R (2001). How does infection with parthenogenesis-inducing Wolbachia reduce the fitness of Trichogramma? J Invertebr Pathol, 78, 267-271.

https://doi.org/10.1006/jipa.2002.5080

Teixeira L, Ferreira Á, Ashburner M (2008). The bacterial symbiont Wolbachia induces resistance to RNA viral infections in Drosophila melanogaster. PLoS Biol, 6, 2753-2763.

https://doi.org/10.1371/journal.pbio.1000002

Turelli M (1994). Evolution of Incompatibility-Inducing Microbes and Their Hosts. Evolution ( $N$ Y), 48, 1500-1513.

https://doi.org/10.1111/j.1558-5646.1994.tb02192.x

Turley AP, Moreira LA, O’Neill SL, McGraw EA (2009). Wolbachia infection reduces bloodfeeding success in the dengue fever mosquito, Aedes aegypti. PLoS Negl Trop Dis, 3, 1-6. https://doi.org/10.1371/journal.pntd.0000516

Weeks AR, Turelli M, Harcombe WR, Reynolds KT, Hoffmann AA (2007). From parasite to 
mutualist: Rapid evolution of Wolbachia in natural populations of Drosophila. PLoS Biol, 5, 0997-1005.

742

743

744

745

746

747

748

749

750

751

752

753

754

755

756

757

758

759

760

761

https://doi.org/10.1371/journal.pbio.0050114

Weinert LA, Araujo-Jnr E V., Ahmed MZ, Welch JJ (2015). The incidence of bacterial endosymbionts in terrestrial arthropods. Proc R Soc B Biol Sci, 282.

https://doi.org/10.1098/rspb.2015.0249

Werren JH, Baldo L, Clark ME (2008). Wolbachia: master manipulators of invertebrate biology. Nat Rev Microbiol, 6, 741-51.

https://doi.org/10.1038/nrmicro1969

Whittle M, Barreaux AMG, Bonsall MB, Whittle M (2021). Insect-host control of obligate , intracellular symbiont density. Proc R Soc B, 288.

https://doi.org/10.1098/rspb.2021.1993

Xi Z, Khoo CCH, Dobson SL (2005). Ecology: Wolbachia establishment and invasion in an Aedes Aegypti laboratory population. Science, 310, 326-328.

https://doi.org/10.1126/science.1117607

Zug R, Hammerstein P (2015). Wolbachia and the insect immune system: What reactive oxygen species can tell us about the mechanisms of Wolbachia-host interactions. Front Microbiol, 6, 1-16.

https://doi.org/10.3389/fmicb.2015.01201

Zuk M, Stoehr AM (2002). Immune defense and host life history. Am Nat, 160.

https://doi.org/10.1086/342131

\section{Figure legends}



$w A w B(P U)$ were $n=98, n=95, n=94$ and $n=95$ respectively. $w A w B(P U)$ were $n=73, n=96, n=95$ and $n=95$ respectively.

Statistical significance was tested using Log Rank statistics with $p<0.05$.

\section{Figure 2. Wolbachia infected males show a reduction in the number of copulations}

Males from different Wolbachia infections were mated sequentially until each of them stopped mating. $\mathrm{O}(\mathrm{PU})$ could perform the highest number of copulations while $w \mathrm{~B}(\mathrm{PU})$ the least. The copulations a male can perform. The figure also shows whether the progenies of these

773 sequential copulations produce any daughters or not, as a measure of sperm depletion. The

774 details of sperm depletion are shown in figure 3. Sample sizes for the strains 0(PU), wA(PU), 775 $0(w A P U), w B(P U), 0(w B P U), w A w B(P U)$ and $0(w A w B P U)$ were $n=7, n=7, n=7, n=6, n=5, n=6$ 776 and $n=7$ respectively.

\section{Figure 3. Wolbachia infected males deplete their sperm faster than the uninfected males}

778 The Y-axis in black on the left of each figure represents the percentage of daughters produced 779 for each mating. The black dots represent the average number of daughters produced for each 780 sequential mating by the males of different Wolbachia infections (detailed in figure 2). The 781 number of daughters produced is taken as a measure of the number of sperm transferred 
782 during each mating. The $\mathrm{Y}$-axis, in grey, on the right, for each figure tallies the average number

783 of copulations that yielded at least one daughter. Thus, it measures the number of mating 784 before a male is depleted of its sperm. The left panel shows the males from Wolbachia infected 785 strains whereas the right panel shows their respective cured versions. Data for $0(P U)$ is 786 repeated at the top for comparison. The statistical significance was tested using the Mann787 Whitney $U$ test with $\mathrm{p}<0.05$

Figure 4. Wolbachia infected females produce fewer offspring

Family size produced by females when hosted as virgins (A) and mated (B). The difference in the family size of mated females is due to the difference in the number of daughters (C) as

791 there is no significant difference in the number of males produced. The statistical significance

792 is tested using the Mann-Whitney U test with $\mathrm{p}<0.05$.

\section{Figure 5. Wolbachia infection reduces female fecundity}

794 The measure of fecundity (number of eggs laid) by females of different infections, with virgin 795 females (A) and mated females (B). For virgin females, O(PU) shows higher fecundity than $796 w \mathrm{~A} w \mathrm{~B}(\mathrm{PU})$, while $w \mathrm{~A}(\mathrm{PU})$ and $w \mathrm{~B}(\mathrm{PU})$ strains have intermediate fecundity which is comparable 797 to both $\mathrm{O}(\mathrm{PU})$ and $w \mathrm{~A} w \mathrm{~B}(\mathrm{PU})$. However, for mated females, $\mathrm{O}(\mathrm{PU})$ and $w \mathrm{~A}(\mathrm{PU})$ show higher 798 fecundity than $w \mathrm{~B}(\mathrm{PU})$ and $w \mathrm{~A} w \mathrm{~B}(\mathrm{PU})$. The statistical significance is tested with the Mann799 Whitney U test, $\mathrm{p}<0.05$. 
800 Figure 6. Quantitative estimation of Wolbachia across different developmental stages of $\boldsymbol{N}$.

801 vitripennis males (A) and females (B). The statistical significance between groups is tested

802 using the Mann-Whitney U test, $\mathrm{p}<0.05$.

803 Table 1. Negative fitness effects of $\mathrm{Cl}$-inducing Wolbachia

804 Table 2. Effect of Wolbachia infections on N. vitripennis (Summary) 
(A)
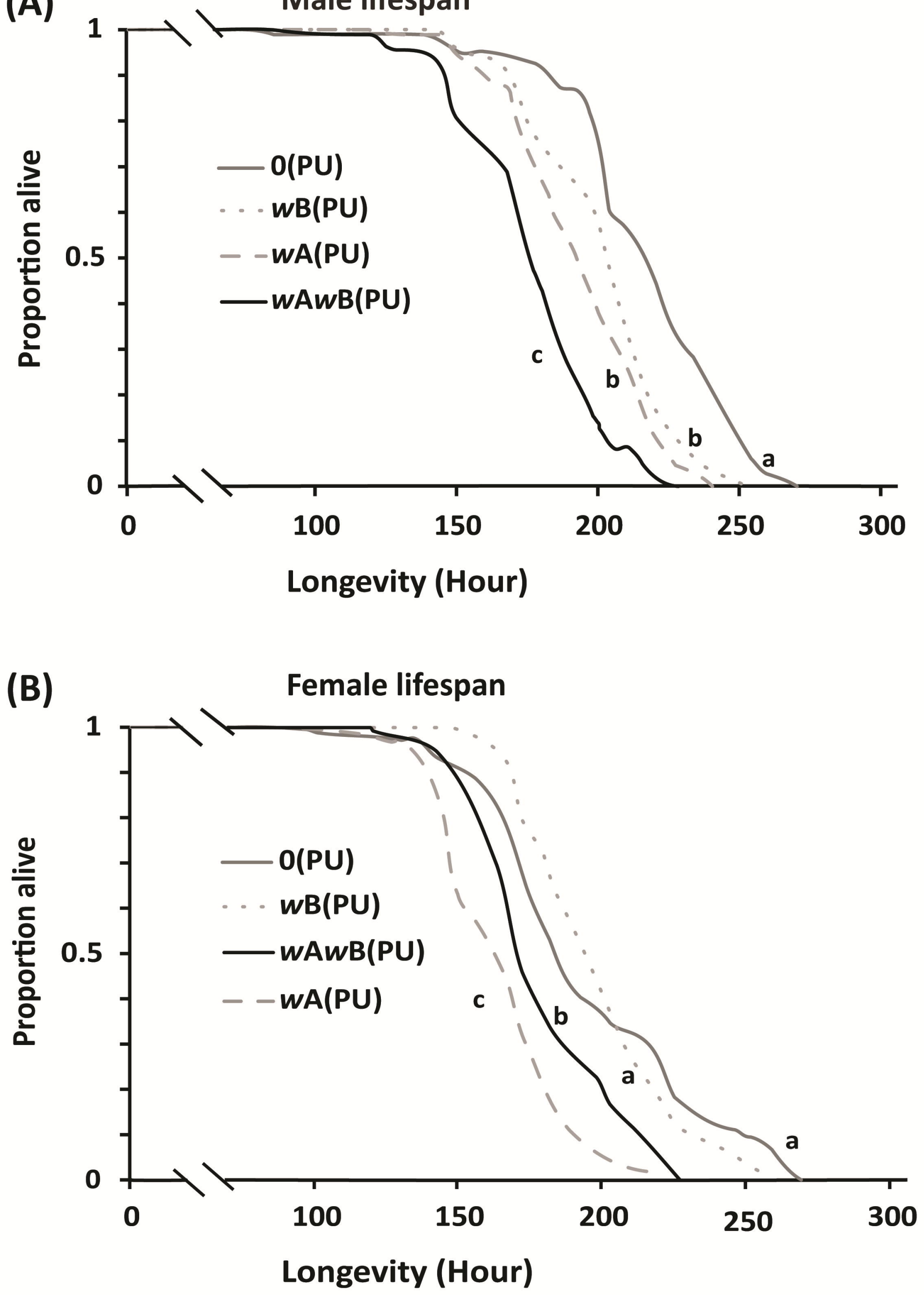
Number of copulations

- Female and male progeny • Only male progeny - No emergence

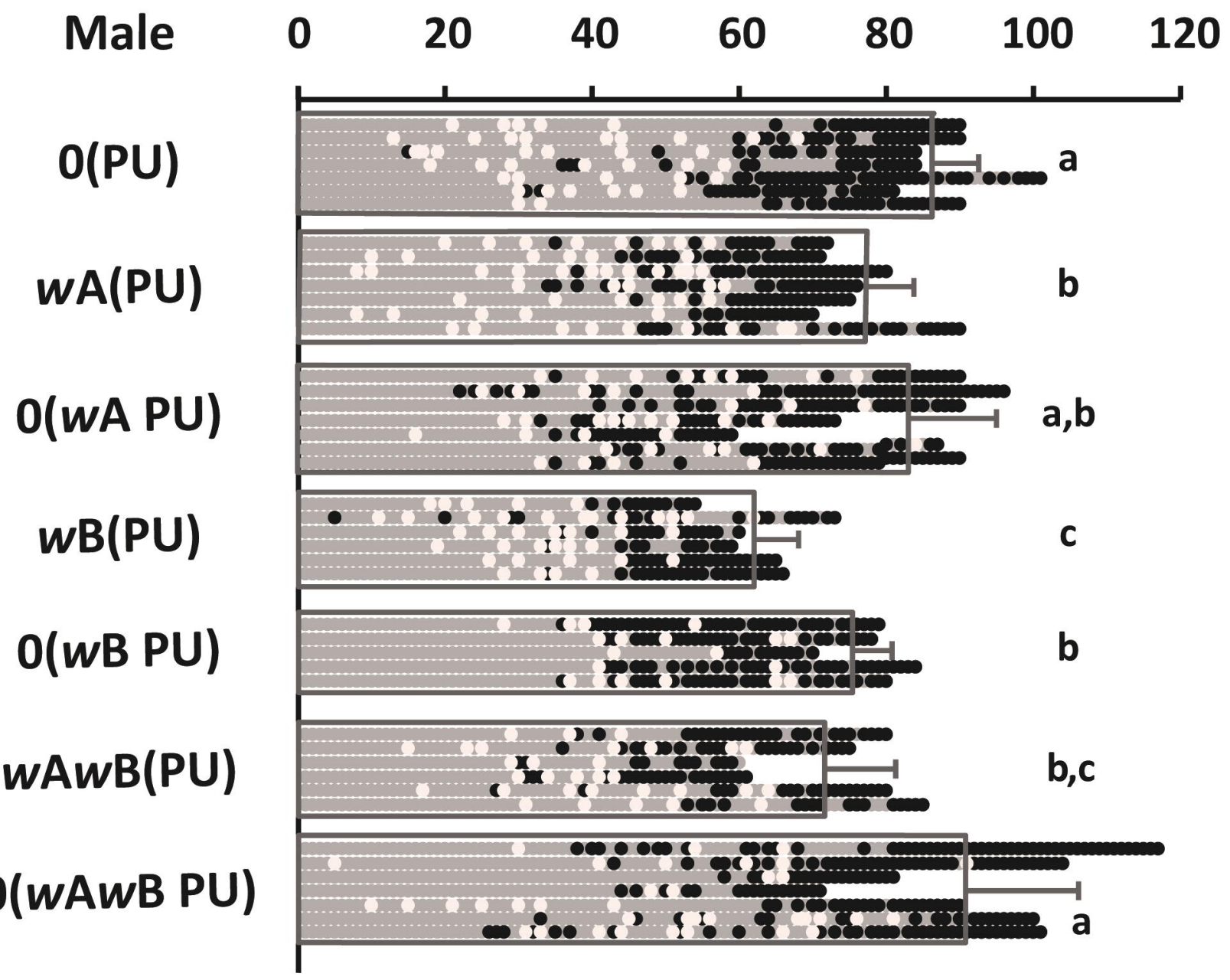



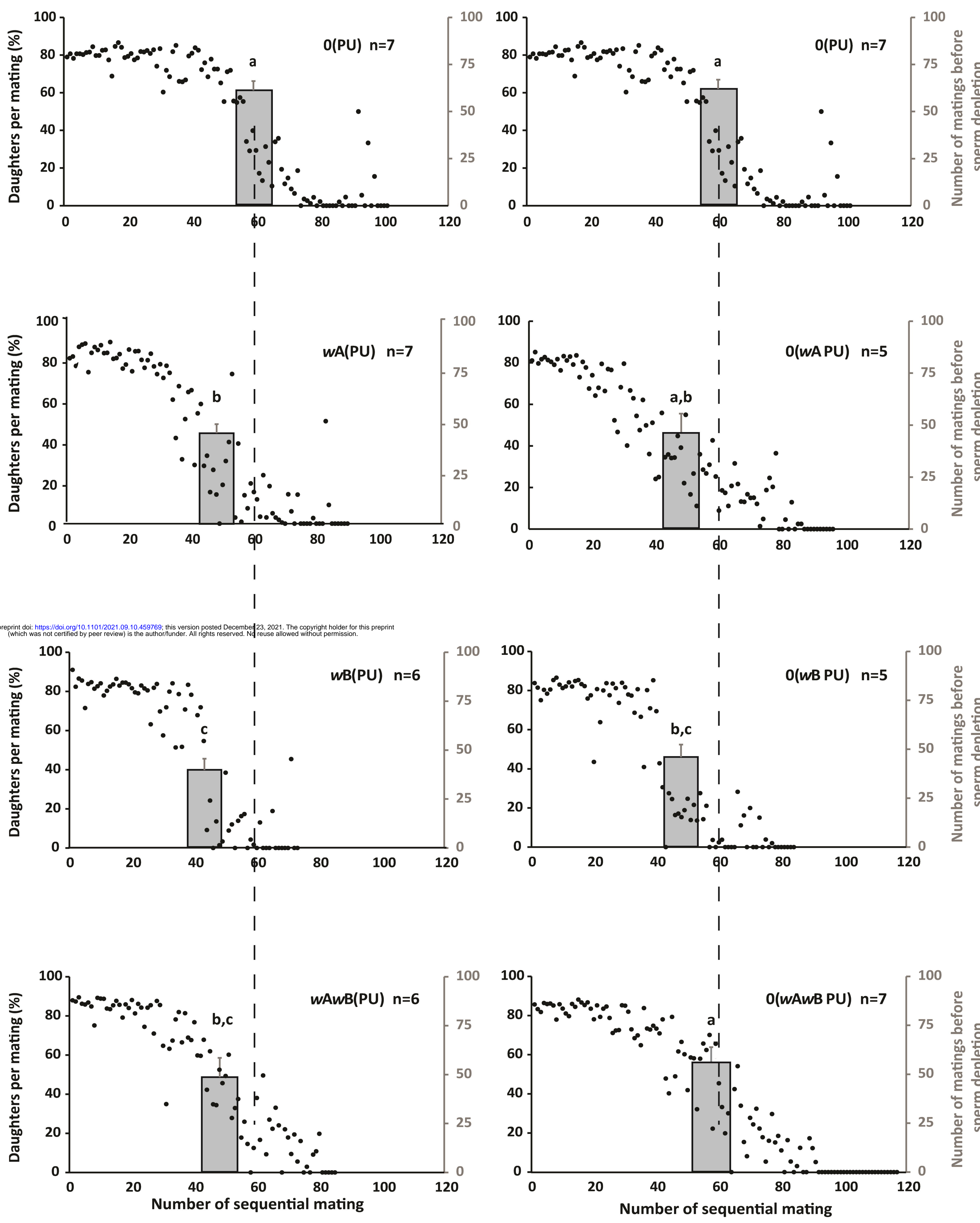


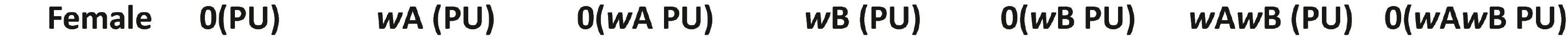
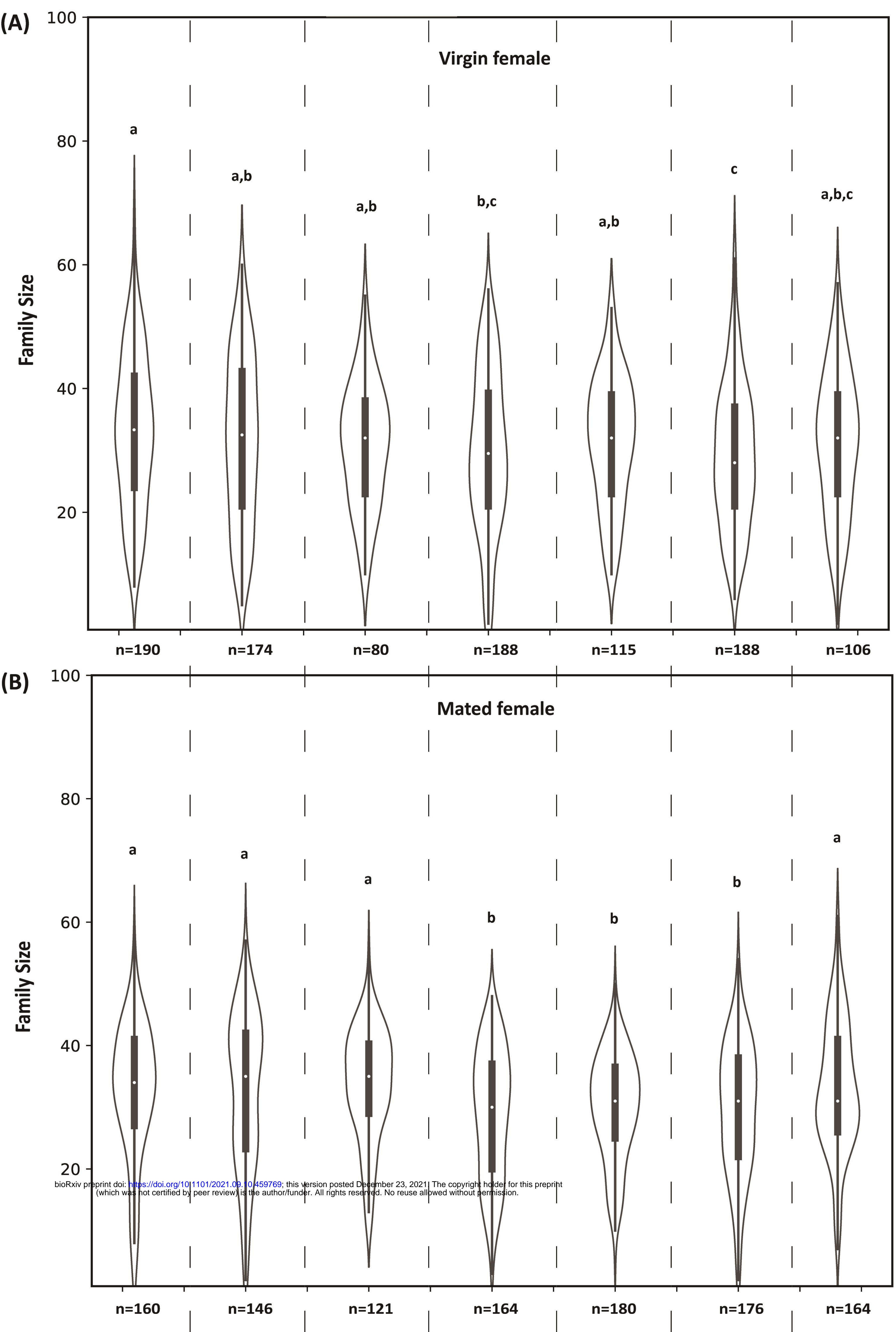

(C)

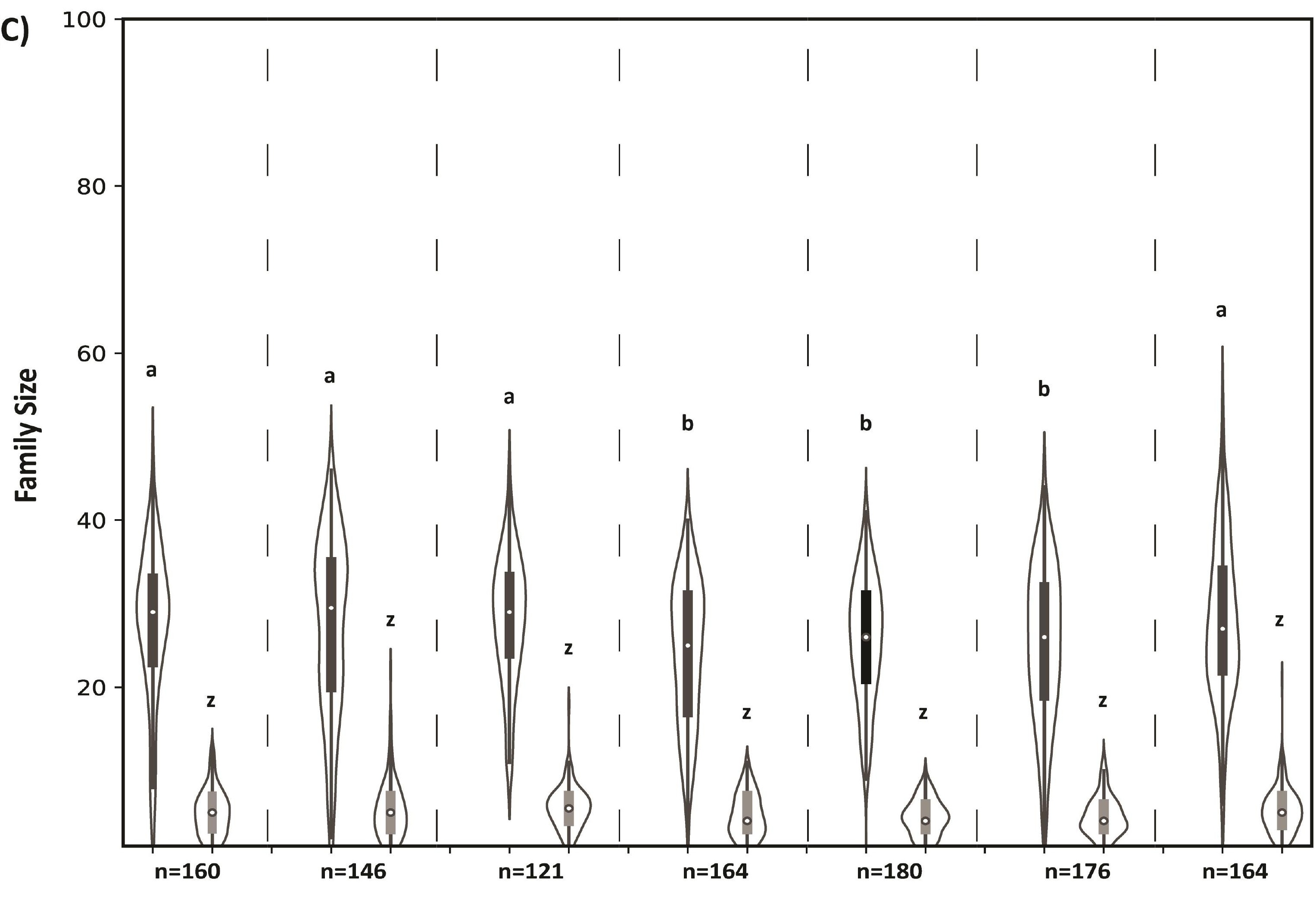


(A) ${ }^{100}$

\section{Virgin Female}

80pio Rxiv prepaht doi: https://doi.org/10.1101/2021.09.10.459769; this version posted December 23, 2021. The copyright holder for this preprint (which was not certified by peer review) is the author/funder. All rights reserved. No reuse allowed without permission.

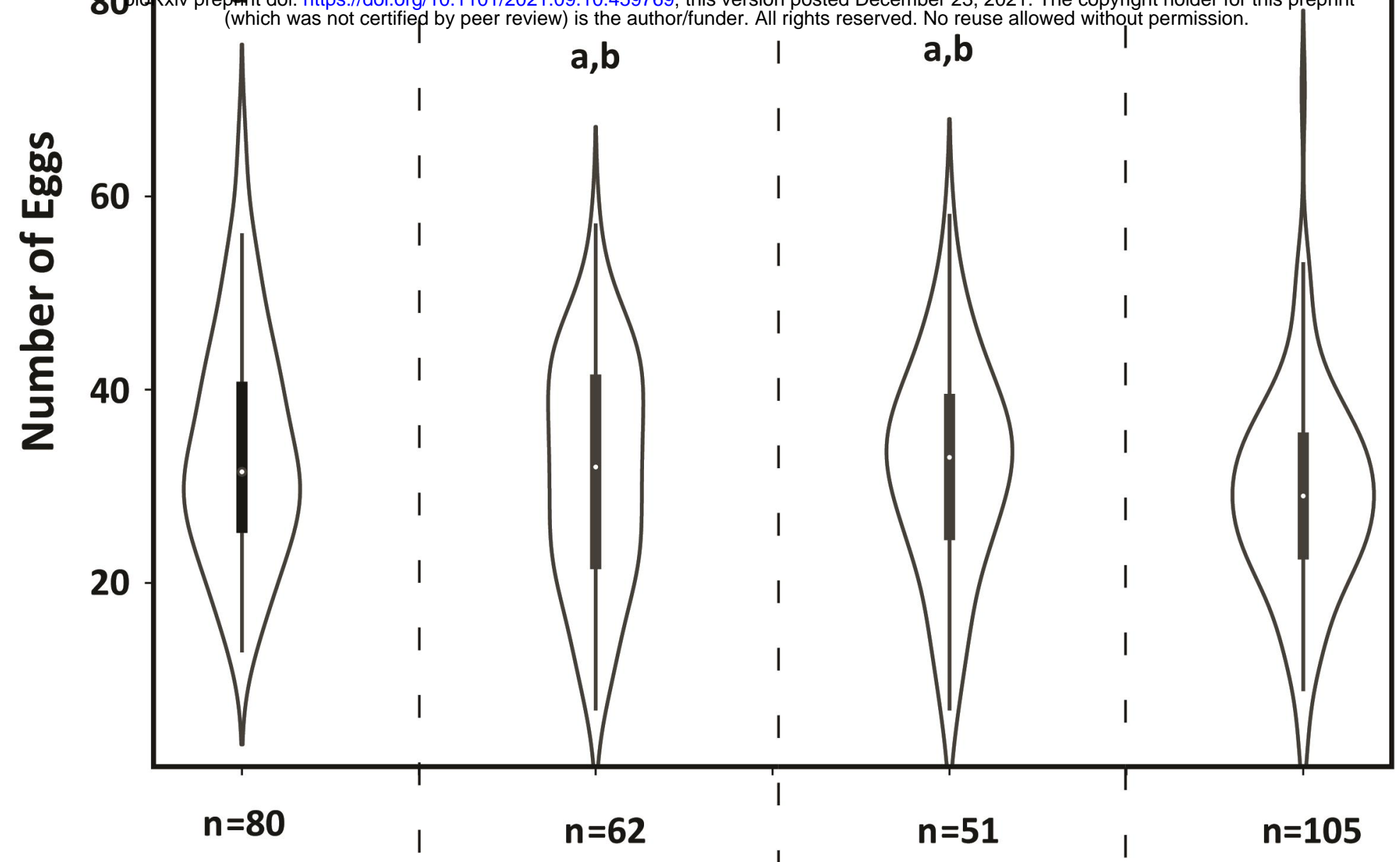

(B)

100

Mated Female

60

80

a

$\mathbf{a}, \mathbf{b}$
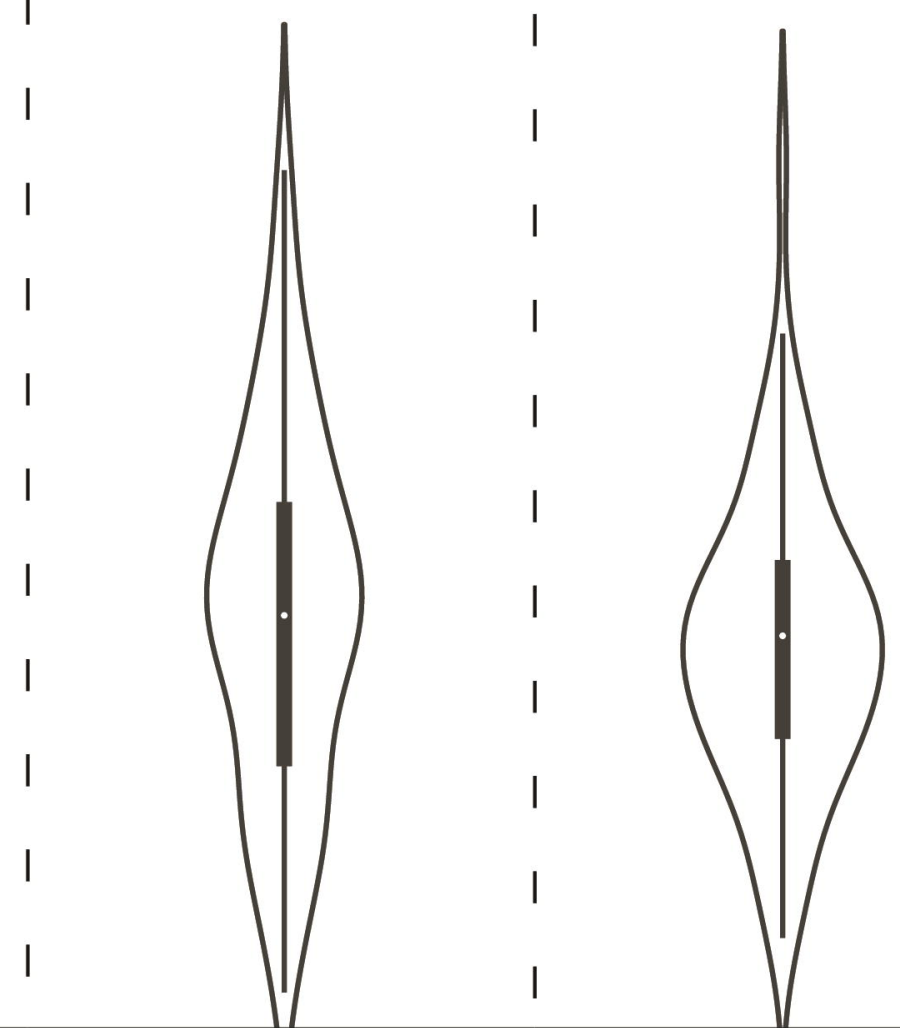

b
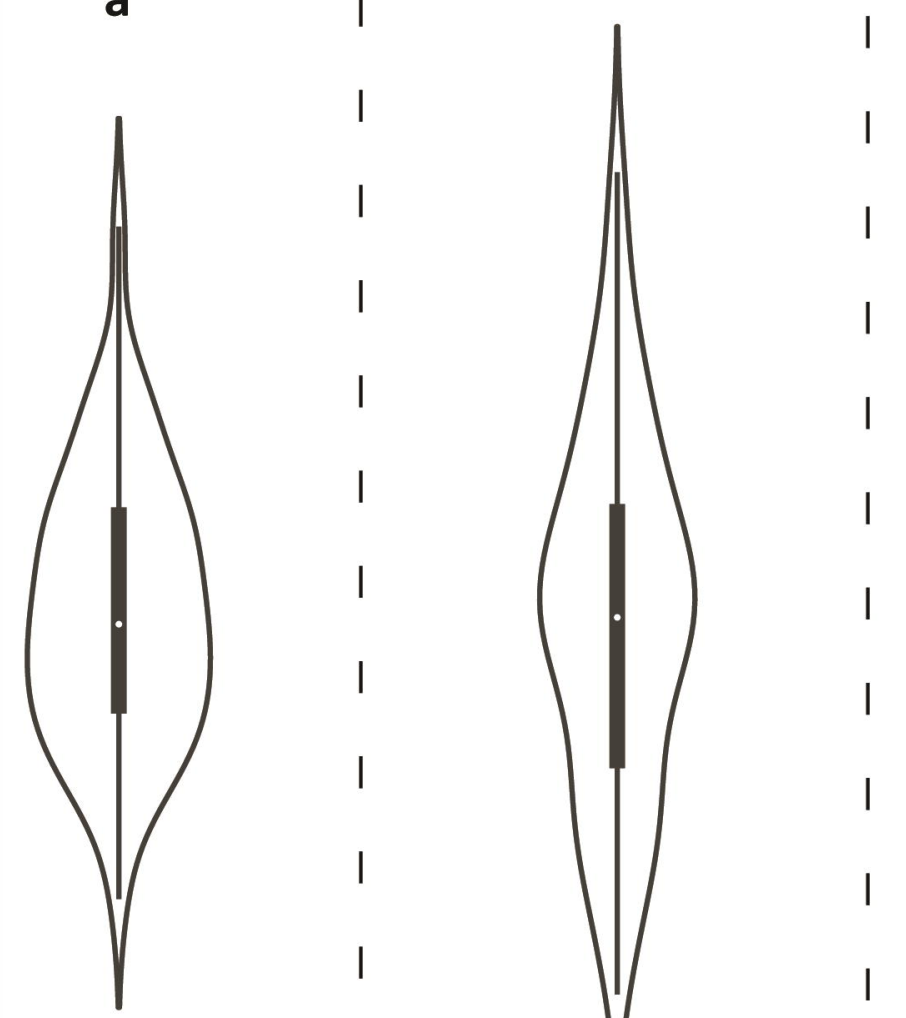
(A) 0.5

Males

0.4

$\underset{\gamma}{\sigma}$

0.3

bioRxiv p eprint doi: https://doi.org/10.1101/2021.09.10.459769; this version posted December 23, 2021. The copyright holder for this preprint (which was not certified by peer review) is the author/funder. All rights reserved. No reuse allowed without permission.

0.2

0.1 .

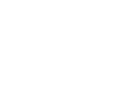


Table 1. Negative fitness effects of $\mathrm{Cl}$-inducing Wolbachia

\begin{tabular}{|c|c|c|c|c|c|c|}
\hline $\begin{array}{l}\text { Wolbachia } \\
\text { Supergroup }\end{array}$ & Genera & Species & Wolbachia Strain & Host Sex & Negative effect & Reference \\
\hline \multirow{11}{*}{ A } & \multirow{11}{*}{ Drosophila } & \multirow{4}{*}{ D. melanogaster } & A-wMelPop & Female/Male & Tissue degeneration, reduced lifespan & Min et al. 1997; Reynolds et al. 2003 \\
\hline & & & A-wMelPop & Female/Male & Decreased response to food cues & Peng et al. 2008 \\
\hline & & & A-wMel & Female & Reduced body size & Hoffmann et al. 1997 \\
\hline & & & A-wMel & Female & $\begin{array}{c}\text { Reduced fecundity after a dormancy } \\
\text { period }\end{array}$ & Kriesner et al. 2016 \\
\hline & & \multirow{6}{*}{ D simulans } & A-wRi & Female & Reduction in fecundity & Hoffmann et al., 1988, 1990 \\
\hline & & & A-wRi & Female & Reduction in fecundity & Snook et al. 2000 \\
\hline & & & $\mathrm{A}-w \mathrm{Ri}$ & Male & Lesser sperm cysts, reduced fertility & Snook et al. 2000 \\
\hline & & & A-wHa & Female & Reduction in fecundity & Fytrou et al. 2006 \\
\hline & & & $\mathrm{A}-w \mathrm{Ha}$ & Female/Male & $\begin{array}{c}\text { Reduced thorax length, reduction in an } \\
\text { immune response against parasitoid } \\
\text { infection }\end{array}$ & Fytrou et al. 2006 \\
\hline & & & $\mathrm{A}-w \mathrm{Ri}$ & Male & Reduced sperm competition & Crespigny et al. 2006 \\
\hline & & D. suzukii & A-wSuz & Female & Reduced progeny family size & Hamm et al. 2014 \\
\hline \multirow{6}{*}{ B } & \multirow{4}{*}{ Aedes } & A. albopictus & A-wAlb, B-wAlb & Female & Reduced lifespan, reduction in fecundity & Islam et al. 2006; Sun et al. 2009 \\
\hline & & \multirow{3}{*}{ A. aegypti } & A-wMelPop & Female & Reduced lifespan, reduction in fecundity & Ross et al. 2019 \\
\hline & & & A-wMelPop & Female/Male & Reduced lifespan & Mcmeniman et al. 2009 \\
\hline & & & A-wMelPop & Female & $\begin{array}{l}\text { Reduction in fecundity, } \\
\text { reduced blood-feeding success }\end{array}$ & Turley et al. 2009, 2013; Allman et al. 202 \\
\hline & \multirow[t]{2}{*}{ Culex } & C. pipiens & B-wPip & Female/Male & Embryonic Mortality & Duron et al. 2005 \\
\hline & & $\begin{array}{c}\text { C. } \\
\text { quinquefasciatus }\end{array}$ & B-wPip & Female & Reduced fecundity & Almeida et al. 2011 \\
\hline
\end{tabular}


Table 2. Effect of Wolbachia infections on N. vitripennis (Summary)

\begin{tabular}{|c|c|c|c|}
\hline Phenotype & Host Sex & Effect of Wolbachia & "Cost" compared to 0(PU) \\
\hline \multirow[t]{2}{*}{ Lifespan } & Male & $\mathrm{O}(\mathrm{PU})>w \mathrm{~B}(\mathrm{PU})=w \mathrm{~A}(\mathrm{PU})>w \mathrm{~A} w \mathrm{~B}(\mathrm{PU})$ & $\begin{array}{c}w \mathrm{~A}(\mathrm{PU})=11.1 \%, w \mathrm{~B}(\mathrm{PU})=6.5 \% \\
w \mathrm{~A} w \mathrm{~B}(\mathrm{PU})=15.5 \%\end{array}$ \\
\hline & Female & $\mathrm{O}(\mathrm{PU})=w \mathrm{~B}(\mathrm{PU})>w \mathrm{~A} w \mathrm{~B}(\mathrm{PU})>w \mathrm{~A}(\mathrm{PU})$ & $w A(P U)=17.7 \%, w A w B(P U)=15.5 \%$ \\
\hline Number of copulations & Male & $\begin{array}{c}\mathrm{O}(\mathrm{PU})>w \mathrm{~A}(\mathrm{PU}) w \mathrm{~B}(\mathrm{PU}) \text { and } w \mathrm{~A} w \mathrm{~B}(\mathrm{PU}) \\
w \mathrm{~A}(\mathrm{PU})= \\
w \mathrm{~A} w \mathrm{~B}(\mathrm{PU}), \quad w \mathrm{~A}(\mathrm{PU})>w \mathrm{~B}(\mathrm{PU}), \\
w \mathrm{~A} w \mathrm{~B}(\mathrm{PU})=w \mathrm{~B}(\mathrm{PU})\end{array}$ & $\begin{array}{c}w A(P U)=12.4 \%, w B(P U)=28.8 \% \\
w A w B(P U)=16.7 \%\end{array}$ \\
\hline Sperm depletion & Male & $\begin{array}{c}\mathrm{O}(\mathrm{PU})>w \mathrm{~A}(\mathrm{PU}) w \mathrm{~B}(\mathrm{PU}) \text { and } w \mathrm{~A} w \mathrm{~B}(\mathrm{PU}) \\
w \mathrm{~A}(\mathrm{PU})= \\
w \mathrm{~A} w \mathrm{~B}(\mathrm{PU}), \quad w \mathrm{~A}(\mathrm{PU})>w \mathrm{~B}(\mathrm{PU}), \\
w \mathrm{~A} w \mathrm{~B}(\mathrm{PU})=w \mathrm{~B}(\mathrm{PU})\end{array}$ & $\begin{array}{c}w \mathrm{~A}(\mathrm{PU})=19.4 \%, w \mathrm{~B}(\mathrm{PU})=31.3 \% \\
w \mathrm{~A} w \mathrm{~B}(\mathrm{PU})=15.7 \%\end{array}$ \\
\hline \multirow{3}{*}{ Progeny family size } & Female & & \\
\hline & a. Virgin & $\begin{array}{c}\mathrm{O}(\mathrm{PU})>w \mathrm{~B}(\mathrm{PU}) \text { and } w \mathrm{~A} w \mathrm{~B}(\mathrm{PU}), \mathrm{O}(\mathrm{PU})=w \mathrm{~A}(\mathrm{PU}) \\
w \mathrm{~A}(\mathrm{PU})> \\
w \mathrm{~A} w \mathrm{~B}(\mathrm{PU}), w \mathrm{~A}(\mathrm{PU})=w \mathrm{~B}(\mathrm{PU}) \\
w \mathrm{~B}(\mathrm{PU})=w \mathrm{~A} w \mathrm{~B}(\mathrm{PU})\end{array}$ & $w \mathrm{~B}(\mathrm{PU})=10 \%, w A w \mathrm{~B}(\mathrm{PU})=12.4 \%$ \\
\hline & b. Mated & $\mathrm{O}(\mathrm{PU})=w \mathrm{~A}(\mathrm{PU})>w \mathrm{~A} w \mathrm{~B}(\mathrm{PU})=w \mathrm{~B}(\mathrm{PU})$ & $w \mathrm{~B}(\mathrm{PU})=11.5 \%, w \mathrm{~A} w \mathrm{~B}(\mathrm{PU})=9.1 \%$ \\
\hline \multirow{3}{*}{ Fecundity } & Female & & \\
\hline & a. Virgin & $\mathrm{O}(\mathrm{PU})=w \mathrm{~A}(\mathrm{PU})=w \mathrm{~B}(\mathrm{PU}), \mathrm{O}(\mathrm{PU})>w \mathrm{~A} w \mathrm{~B}(\mathrm{PU})$ & $w A w B(P U)=14.1 \%$ \\
\hline & b. Mated & $\mathrm{O}(\mathrm{PU})=w \mathrm{~A}(\mathrm{PU})>w \mathrm{~B}(\mathrm{PU})=w \mathrm{~A} w \mathrm{~B}(\mathrm{PU})$ & $w \mathrm{~B}(\mathrm{PU})=9.1 \%, w \mathrm{~A} w \mathrm{~B}(\mathrm{PU})=18.2 \%$ \\
\hline \multirow{2}{*}{ Wolbachia density } & Male & $w A w B(P U)>w B(P U)=w A(P U)$ & \\
\hline & Female & $w A w B(P U)>w A(P U)=w B(P U)$ & \\
\hline
\end{tabular}

